\title{
Equilibrium of Bayesian fuzzy economies and quasi-variational inequalities with random fuzzy mappings
}

Monica Patriche*

"Correspondence:

monica.patriche@yahoo.com Department of Mathematics, University of Bucharest, 14 Academiei Street, Bucharest, 010014, Romania

\begin{abstract}
In this paper, we introduce a Bayesian abstract fuzzy economy model, and we prove the existence of Bayesian fuzzy equilibrium. As applications, we prove the existence of the solutions for two types of random quasi-variational inequalities with random fuzzy mappings, and we also obtain random fixed point theorems.

MSC: 58E35; 47H10; 91B50; 91A44

Keywords: Bayesian abstract fuzzy economy; Bayesian fuzzy equilibrium; incomplete information; random fixed point; random quasi-variational inequalities; random fuzzy mapping
\end{abstract}

\section{Introduction}

The study of fuzzy games began with the paper written by Kim and Lee in 1998 [1]. This type of games is a generalization of classical abstract economies. For an overview of results concerning this topic, the reader is referred to [2]. However, the existence of random fuzzy equilibrium has not been studied so far. We introduce the new model of Bayesian abstract fuzzy economy, and we explore the existence of the Bayesian fuzzy equilibrium. Our model is characterized by a private information set, an action (strategy) fuzzy mapping, a random fuzzy constraint one and a random fuzzy preference mapping. The Bayesian fuzzy equilibrium concept is an extension of the deterministic equilibrium. We generalize the former deterministic models introduced by Debreu [3], Shafer and Sonnenschein [4], Yannelis and Prabhakar [5] or Patriche [2], and we search for applications.

Since Fichera and Stampacchia introduced the variational inequalities (in 1960s), this domain has been extensively studied. For recent results we refer the reader to [6-11] and the bibliography therein. Noor and Elsanousi [12] introduced the notion of a random variational inequality. The existence of solutions of the random variational inequality and random quasi-variational inequality problems has been proved, for instance, in [13-21].

In this paper, we first define the model of the Bayesian abstract fuzzy economy and we prove a theorem of Bayesian fuzzy equilibrium existence. Then, we apply it in order to prove the existence of solutions for the two types of random quasi-variational inequalities with random fuzzy mappings. We generalize some results obtained by Yuan in [22]. As a consequence, we obtain random fixed point theorems.

The paper is organized as follows. In the next section, some notational and terminological conventions are given. We also present, for the reader's convenience, some results

\section{Springer}

(c) 2013 Patriche; licensee Springer. This is an Open Access article distributed under the terms of the Creative Commons Attribution License (http://creativecommons.org/licenses/by/2.0), which permits unrestricted use, distribution, and reproduction in any medium, provided the original work is properly cited. 
on Bochner integration. In Section 3, the model of differential information abstract fuzzy economy is introduced, and the main result is also stated. Section 4 contains existence results for solutions of random quasi-variational inequalities with random fuzzy mappings.

\section{Notation and definition}

Throughout this paper, we shall use the following notation.

$\mathbb{R}_{++}$denotes the set of strictly positive reals. co $D$ denotes the convex hull of the set $D$. $\overline{\mathrm{co}} D$ denotes the closed convex hull of the set $D .2^{D}$ denotes the set of all nonempty subsets of the set $D$. If $D \subset Y$, where $Y$ is a topological space, $\operatorname{cl} D$ denotes the closure of $D$.

For the reader's convenience, we review a few basic definitions and results from continuity and measurability of correspondences and Bochner integrable functions.

Let $Z$ and $Y$ be sets. Let $Z, Y$ be topological spaces and $P: Z \rightarrow 2^{Y}$ be a correspondence. $P$ is said to be upper semicontinuous if for each $z \in Z$ and each open set $V$ in $Y$ with $P(z) \subset V$, there exists an open neighborhood $U$ of $z$ in $Z$ such that $P(y) \subset V$ for each $y \in U$. $P$ is said to be lower semicontinuous if for each $z \in Z$ and each open set $V$ in $Y$ with $P(z) \cap V \neq \emptyset$, there exists an open neighborhood $U$ of $z$ in $Z$ such that $P(y) \cap V \neq \emptyset$ for each $y \in U$.

Lemma 1 [22] Let $Z$ and $Y$ be two topological spaces, and let $D$ be an open subset of $Z$. Suppose $P_{1}: Z \rightarrow 2^{Y}, P_{2}: Z \rightarrow 2^{Y}$ are upper semicontinuous correspondences such that $P_{2}(z) \subset P_{1}(z)$ for all $z \in D$. Then the correspondence $P: Z \rightarrow 2^{Y}$ defined by

$$
P(z)= \begin{cases}P_{1}(z) & \text { if } z \notin D ; \\ P_{2}(z) & \text { if } z \in D\end{cases}
$$

is also upper semicontinuous.

Let $E$ be a topological vector space, and let $E^{\prime}$ be the dual space of $E$, which consists of all continuous linear functionals on $E$. The real part of pairing between $E^{\prime}$ and $E$ is denoted by $\operatorname{Re}\langle w, x\rangle$ for each $w \in E^{\prime}$ and $x \in E$. The operator $P: E \rightarrow 2^{E^{\prime}}$ is called monotone if $\operatorname{Re}\langle u-v, y-x\rangle \geq 0$ for all $u \in P(y)$ and $v \in P(x)$ and $x, y \in E$.

Let now $(\Omega, \mathcal{F}, \mu)$ be a complete, finite measure space, and $Y$ be a topological space. The correspondence $P: \Omega \rightarrow 2^{Y}$ is said to have a measurable graph if $G_{P} \in \mathcal{F} \otimes \beta(Y)$, where $\beta(Y)$ denotes the Borel $\sigma$-algebra on $Y$ and $\otimes$ denotes the product $\sigma$-algebra. The correspondence $T: \Omega \rightarrow 2^{Y}$ is said to be lower measurable if for every open subset $V$ of $Y$, the set $\{\omega \in \Omega: T(\omega) \cap V \neq \emptyset\}$ is an element of $\mathcal{F}$. This notion of measurability is also called in the literature weak measurability or just measurability, in comparison with the strong measurability: the correspondence $T: \Omega \rightarrow 2^{Y}$ is said to be strong measurable if for every closed subset $V$ of $Y$, the set $\{\omega \in \Omega: T(\omega) \cap V \neq \emptyset\}$ is an element of $\mathcal{F}$. In the framework we shall deal with (complete finite measure spaces), the two notions coincide (see [23]).

Recall (see Debreu [24], p.359) that if $T: \Omega \rightarrow 2^{Y}$ has a measurable graph, then $T$ is lower measurable. Furthermore, if $T(\cdot)$ is closed-valued and lower measurable, then $T$ : $\Omega \rightarrow 2^{Y}$ has a measurable graph.

Lemma 2 [25] Let $P_{n}: \Omega \rightarrow 2^{Y}, n=1,2 \ldots$ be a sequence of correspondences with measurable graphs. Then the correspondences $\bigcup_{n} P_{n}, \bigcap_{n} P_{n}$ and $Y \backslash P_{n}$ have measurable graphs. 
Let $(\Omega, \mathcal{F}, \mu)$ be a measure space, and let $Y$ be a Banach space.

It is known (see [25], Theorem 2, p.45) that if $x: \Omega \rightarrow Y$ is a $\mu$-measurable function, then $x$ is the Bochner integrable if and only if $\int_{\Omega}\|x(\omega)\| d \mu(\omega)<\infty$.

It is denoted by $L_{1}(\mu, Y)$, the space of equivalence classes of $Y$-valued Bochner integrable functions $x: \Omega \rightarrow Y$ normed by $\|x\|=\int_{\Omega}\|x(\omega)\| d \mu(\omega)$. Also, it is known (see [24], p.50) that $L_{1}(\mu, Y)$ is a Banach space.

The correspondence $P: \Omega \rightarrow 2^{Y}$ is said to be integrably bounded if there exists a map $h \in L_{1}(\mu, R)$ such that $\sup \{\|x\|: x \in P(\omega)\} \leq h(\omega) \mu$-a.e.

We denote by $S_{P}$ the set of all selections of the correspondence $P: \Omega \rightarrow 2^{Y}$ that belong to the space $L_{1}(\mu, Y)$, i.e.,

$$
S_{P}=\left\{x \in L_{1}(\mu, Y): x(\omega) \in P(\omega) \mu \text {-a.e. }\right\} .
$$

We will find the conditions under which $S_{P}$ is nonempty and weakly compact in $L_{1}(\mu, Y)$ by applying Aumann measurable selection theorem (see Appendix) and Diestel's theorem (see Appendix).

Zadeh initiated the theory of fuzzy sets [26] as a framework for phenomena, which can not be characterized precisely. We present below several notions concerning the fuzzy sets and the fuzzy mappings.

Definition 1 (Chang [27]) If $Y$ is a topological space, then a function $A$ from $Y$ into [0;1] is called a fuzzy set on $Y$. The family of all fuzzy sets on $Y$ is denoted by $\mathcal{F}(Y)$.

(2) If $X$ and $Y$ are topological spaces, then a mapping $P: X \rightarrow \mathcal{F}(Y)$ is called a fuzzy mapping.

(3) If $P$ is a fuzzy mapping, then, for each $x \in X, P(x)$ is a fuzzy set in $Y$ and $P(x)(y) \in[0,1], y \in Y$ is called the degree of membership of $y$ in $P(x)$.

(4) Let $A \in \mathcal{F}(Y), a \in[0,1]$, then the set $(A)_{a}=\{y \in Y: A(y)>a\}$ is called a strong $a$-cut set of the fuzzy set $A$.

The random fuzzy mappings have been defined in order to model random mechanisms generating imprecisely-valued data which can be properly described by using fuzzy sets.

Let $Y$ be a topological space, let $\mathcal{F}(Y)$ be a collection of all fuzzy sets over $Y$, and let $(\Omega, \mathcal{F})$ be a measurable space.

Definition 2 (See [28]) A fuzzy mapping $P: \Omega \rightarrow \mathcal{F}(Y)$ is said to be measurable if for any given $a \in[0,1],(P(\cdot))_{a}: \Omega \rightarrow 2^{Y}$ is a measurable set-valued mapping.

(2) We say that a fuzzy mapping $P: \Omega \rightarrow \mathcal{F}(Y)$ is said to have a measurable graph if for any given $a \in[0,1]$, the set-valued mapping $(P(\cdot))_{a}: \Omega \rightarrow 2^{Y}$ has a measurable graph.

(3) A fuzzy mapping $P: \Omega \times X \rightarrow \mathcal{F}(Y)$ is called a random fuzzy mapping if, for any given $x \in X, P(\cdot, x): \Omega \rightarrow \mathcal{F}(Y)$ is a measurable fuzzy mapping.

\section{Bayesian fuzzy equilibrium existence for Bayesian abstract fuzzy economies}

\subsection{The model of a Bayesian abstract fuzzy economy}

The framework of fuzziness became part of the language of applied mathematics. The uncertainties characterize the individual feature of the decisions of the agents involved in different economic activities, and they can be described by using random fuzzy mappings. 
In the fuzzy model of the abstract economy, which we will define below, for each agent $i$, the action choice is modelled by the measurable fuzzy mapping $X_{i}$, and the constraints and the preferences are modelled by the random fuzzy mappings $A_{i}$ and, respectively, $P_{i}$. In the state of the world $\omega \in \Omega$, the interpretation of the number $P_{i}(\omega, \widetilde{x})(y) \in[0,1]$, associated with $\left(\widetilde{x}_{i}(\omega), y\right)$, can be the degree of intensity, with which $y$ is preferred to $\tilde{x}_{i}(\omega)$, or the degree of truth that $y$ is preferred to $\tilde{x}_{i}(\omega)$. We can also see the value $A_{i}(\omega, \widetilde{x})(y) \in[0,1]$ associated with $\left(\widetilde{x}_{i}(\omega), y\right)$, as the belief of the player $i$ that in the state $\omega$, if the other players choose $\left(\widetilde{x}_{j}(\omega)\right)_{j \neq i}$, he can choose $y \in Y$. The element $z_{i}$ is the action level in each state of the world, $a_{i}(\widetilde{x})$ expresses the perceived degree of feasibility of the strategy $\widetilde{x}$, and $p_{i}(\widetilde{x})$ represents the preference level of the strategy $\tilde{x}$.

We now define the next model of the Bayesian abstract fuzzy economy, which generalizes the model in [29].

Let $(\Omega, \mathcal{F}, \mu)$ be a complete finite measure space, where $\Omega$ denotes the set of states of nature of the world, and the $\sigma$-algebra $\mathcal{F}$ denotes the set of events. Let $Y$ denote the strategy or commodity space, where $Y$ is a separable Banach space.

Let $I$ be a countable or uncountable set (the set of agents). For each $i \in I$, let $X_{i}: \Omega \rightarrow$ $\mathcal{F}(Y)$ be a fuzzy mapping, and let $z_{i} \in(0,1]$.

Let $L_{X_{i}}=\left\{x_{i} \in S_{\left(X_{i}(\cdot)\right)_{z_{i}}: x_{i}}\right.$ is $\mathcal{F}_{i}$-measurable $\}$. Denote by $L_{X}=\prod_{i \in I} L_{X_{i}}$ and by $L_{X_{-i}}$ the set $\prod_{j \neq i} L_{X_{j}}$. An element $x_{i}$ of $L_{X_{i}}$ is called a strategy for agent $i$. The typical element of $L_{X_{i}}$ is denoted by $\tilde{x}_{i}$ and that of $\left(X_{i}(\omega)\right)_{z_{i}}$ by $x_{i}(\omega)$ (or $\left.x_{i}\right)$.

Definition 3 A general Bayesian abstract fuzzy economy is a family $G=\left\{(\Omega, \mathcal{F}, \mu),\left(X_{i}, \mathcal{F}_{i}\right.\right.$, $\left.\left.A_{i}, P_{i}, a_{i}, b_{i}, z_{i}\right)_{i \in I}\right\}$, where

(a) $X_{i}: \Omega \rightarrow \mathcal{F}(Y)$ is the action (strategy) fuzzy mapping of agent $i$;

(b) $\mathcal{F}_{i}$ is a sub $\sigma$-algebra of $\mathcal{F}$, which denotes the private information of agent $i$;

(c) for each $\omega \in \Omega, A_{i}(\omega, \cdot): L_{X} \rightarrow \mathcal{F}(Y)$ is the random fuzzy constraint mapping of agent $i$

(d) for each $\omega \in \Omega, P_{i}(\omega, \cdot): L_{X} \rightarrow \mathcal{F}(Y)$ is the random fuzzy preference mapping of agent $i$

(e) $a_{i}: L_{X} \rightarrow(0,1]$ is a random fuzzy constraint function, and $p_{i}: L_{X} \rightarrow(0,1]$ is a random fuzzy preference function of agent $i$;

(f) $z_{i} \in(0,1]$ is such that for all $(\omega, x) \in \Omega \times L_{X},\left(A_{i}(\omega, \widetilde{x})\right)_{a_{i}(\tilde{x})} \subset\left(X_{i}(\omega)\right)_{z_{i}}$ and $\left(P_{i}(\omega, \widetilde{x})\right)_{p_{i}(\widetilde{x})} \subset\left(X_{i}(\omega)\right)_{z_{i}}$.

Definition 4 A Bayesian fuzzy equilibrium for $G$ is a strategy profile $\widetilde{x}^{*} \in L_{X}$ such that for all $i \in I$,

(i) $\widetilde{x}_{i}^{*}(\omega) \in \operatorname{cl}\left(A_{i}\left(\omega, \widetilde{x}^{*}\right)\right)_{a_{i}\left(\tilde{x}^{*}\right)} \mu$-a.e.;

(ii) $\left(A_{i}\left(\omega, \widetilde{x}^{*}\right)\right)_{a_{i}\left(\tilde{x}^{*}\right)} \cap\left(P_{i}\left(\omega, \widetilde{x}^{*}\right)\right)_{p_{i}\left(\tilde{x}^{*}\right)}=\emptyset \mu$-a.e.

Remark 1 If the correspondences from the model above are constant with respect to $\Omega$, we obtain the abstract fuzzy economy model.

\subsection{Existence of the Bayesian fuzzy equilibrium}

This is our first theorem. The constraint and preference correspondences, derived from the constraint and preference fuzzy mappings, verify the assumptions of measurable graph and weakly open lower sections. Our result is a generalization of Theorem 3 in [29]. 
Theorem 1 Let I be a countable or uncountable set. Let the family $G=\left\{(\Omega, \mathcal{F}, \mu),\left(X_{i}, \mathcal{F}_{i}\right.\right.$, $\left.\left.A_{i}, P_{i}, a_{i}, b_{i}, z_{i}\right)_{i \in I}\right\}$ be a general Bayesian abstract economy satisfying (A.1)-(A.4). Then there exists a Bayesian fuzzy equilibrium for $G$.

For each $i \in I$ :

(A.1) (a) $X_{i}: \Omega \rightarrow \mathcal{F}(Y)$ is such that $\omega \rightarrow X_{i}(\omega)_{z_{i}}: \Omega \rightarrow 2^{Y}$ is a nonempty convex weakly compact-valued and integrably bounded correspondence;

(b) $X_{i}: \Omega \rightarrow \mathcal{F}(Y)$ is such that $\omega \rightarrow\left(X_{i}(\omega)\right)_{z_{i}}: \Omega \rightarrow 2^{Y}$ is $\mathcal{F}_{i}$-lower measurable;

(A.2) (a) For each $(\omega, \widetilde{x}) \in \Omega \times L_{X},\left(A_{i}(\omega, \widetilde{x})\right)_{a_{i}(\tilde{x})}$ is convex and has a nonempty interior in the relative norm topology of $\left(X_{i}(\omega)\right)_{z_{i}}$;

(b) the correspondence $(\omega, \widetilde{x}) \rightarrow\left(A_{i}(\omega, \widetilde{x})\right)_{a_{i}(\widetilde{x})}: \Omega \times L_{X} \rightarrow 2^{Y}$ has a measurable graph, i.e., $\left\{(\omega, \tilde{x}, y) \in \Omega \times L_{X} \times Y: y \in\left(A_{i}(\omega, \widetilde{x})\right)_{a_{i}(\tilde{x})}\right\} \in \mathcal{F} \otimes \beta_{w}\left(L_{X}\right) \otimes \beta(Y)$, where $\beta_{w}\left(L_{X}\right)$ is the Borel $\sigma$-algebra for the weak topology on $L_{X}$ and $\beta(Y)$ is the Borel $\sigma$-algebra for the norm topology on $Y$;

(c) the correspondence $(\omega, \widetilde{x}) \rightarrow\left(A_{i}(\omega, \widetilde{x})\right)_{a_{i}(\widetilde{x})}$ has weakly open lower sections, i.e., for each $\omega \in \Omega$ and for each $y \in Y$, the set $\left(\left(A_{i}(\omega, \widetilde{x})\right)_{a_{i}(\tilde{x})}\right)^{-1}(\omega, y)=\left\{\tilde{x} \in L_{X}: y \in\left(A_{i}(\omega, \widetilde{x})\right)_{a_{i}(\widetilde{x})}\right\}$ is weakly open in $L_{X} ;$

(d) For each $\omega \in \Omega, \widetilde{x} \rightarrow \operatorname{cl}\left(A_{i}(\omega, \widetilde{x})\right)_{a_{i}(\tilde{x})}: L_{X} \rightarrow 2^{Y}$ is upper semicontinuous in the sense that the set $\left\{\tilde{x} \in L_{X}: \operatorname{cl}\left(A_{i}(\omega, \widetilde{x})\right)_{a_{i}(\tilde{x})} \subset V\right\}$ is weakly open in $L_{X}$ for every norm open subset $V$ of $Y$;

(A.3) (a) the correspondence $(\omega, \widetilde{x}) \rightarrow\left(P_{i}(\omega, \widetilde{x})\right)_{p_{i}(\widetilde{x})}: \Omega \times L_{X} \rightarrow 2^{Y}$ has open convex values such that $\left(P_{i}(\omega, \widetilde{x})\right)_{p_{i}(\widetilde{x})} \subset(X(\omega))_{z_{i}}$ for each $(\omega, \widetilde{x}) \in \Omega \times L_{X}$;

(b) the correspondence $(\omega, \widetilde{x}) \rightarrow\left(P_{i}(\omega, \widetilde{x})\right)_{p_{i}(\tilde{x})}: \Omega \times L_{X} \rightarrow 2^{Y}$ has a measurable graph;

(c) the correspondence $(\omega, \widetilde{x}) \rightarrow\left(P_{i}(\omega, \widetilde{x})\right)_{p_{i}(\widetilde{x})}: \Omega \times L_{X} \rightarrow 2^{Y}$ has weakly open lower sections, i.e., for each $\omega \in \Omega$ and for each $y \in Y$, the set $\left(\left(P_{i}(\omega, \widetilde{x})\right)_{p_{i}(\widetilde{x}}\right)^{-1}(\omega, y)=\left\{\widetilde{x} \in L_{X}: y \in\left(P_{i}(\omega, \widetilde{x})\right)_{p_{i}(\tilde{x})}\right\}$ is weakly open in $L_{X} ;$

(A.4) (a) For each $\tilde{x}_{i} \in L_{X_{i}}$, for each $\omega \in \Omega, \widetilde{x}_{i}(\omega) \notin\left(A_{i}(\omega, \widetilde{x})\right)_{a_{i}(\widetilde{x})} \cap\left(P_{i}(\omega, \widetilde{x})\right)_{p_{i}(\tilde{x})}$.

Proof For each $i \in I$, let us define $\Phi_{i}: \Omega \times L_{X} \rightarrow 2^{Y}$ by $\Phi_{i}(\omega, \widetilde{x})=\left(A_{i}(\omega, \widetilde{x})\right)_{a_{i}(\widetilde{x})} \cap$ $\left(P_{i}(\omega, \widetilde{x})\right)_{p_{i}(\widetilde{x})}$. We prove first that $L_{X}$ is a nonempty convex weakly compact subset in $L_{1}(\mu, Y)$.

Since $(\Omega, \mathcal{F}, \mu)$ is a complete finite measure space, $Y$ is a separable Banach space, and $X_{i}: \Omega \rightarrow 2^{Y}$ has a measurable graph, by Aumann's selection theorem (see Appendix), it follows that there exists a $\mathcal{F}_{i}$-measurable function $f_{i}: \Omega \rightarrow Y$ such that $f_{i}(\omega) \in X_{i}(\omega) \mu$ a.e. Since $X_{i}$ is integrably bounded, we have that $f_{i} \in L_{1}(\mu, Y)$, hence $L_{X_{i}}$ is nonempty and $L_{X}=\prod_{i \in I} L_{X_{i}}$ is nonempty. Obviously, $L_{X_{i}}$ is convex and $L_{X}$ is also convex. Since $X_{i}: \Omega \rightarrow$ $2^{Y}$ is integrably bounded and it has convex weakly compact values, by Diestel's theorem (see Appendix), it follows that $L_{X_{i}}$ is a weakly compact subset of $L_{1}(\mu, Y)$. More over, $L_{X}$ is weakly compact. $L_{1}(\mu, Y)$ equipped with the weak topology is a locally convex topological vector space.

The correspondence $\Phi_{i}$ is convex valued, by Lemma 2 , it has a measurable graph, and for each $\omega \in \Omega, \Phi_{i}(\omega, \cdot)$ has weakly open lower sections. Let $U_{i}=\left\{(\omega, \widetilde{x}) \in \Omega \times L_{X}\right.$ : $\left.\Phi_{i}(\omega, \widetilde{x}) \neq \emptyset\right\}$. For each $\tilde{x} \in L_{X}$, let $U_{i}^{\widetilde{x}}=\left\{\omega \in \Omega: \Phi_{i}(\omega, \widetilde{x}) \neq \emptyset\right\}$ and for each $\omega \in \Omega$, let $U_{i}^{\omega}=\left\{\tilde{x} \in L_{X}: \Phi_{i}(\omega, \tilde{x}) \neq \emptyset\right\}$. The values of $\Phi_{i / U_{i}}$ have nonempty interiors in the relative norm topology of $X_{i}(\omega)$. By the Caratheodory-type selection theorem (see Appendix), there exists a function $f_{i}: U_{i} \rightarrow Y$ such that $f_{i}(\omega, \widetilde{x}) \in \Phi_{i}(\omega, \widetilde{x})$ for all $(\omega, \widetilde{x}) \in U_{i}$, for each 
$\tilde{x} \in L_{X}, f_{i}(\cdot, \widetilde{x})$ is measurable on $U_{i}^{\widetilde{x}}$, for each $\omega \in \Omega, f_{i}(\omega, \cdot)$ is continuous on $U_{i}^{\omega}$ and, moreover $f_{i}(\cdot, \cdot)$ is jointly measurable.

Define $G_{i}: \Omega \times L_{X} \rightarrow 2^{Y}$ by $G_{i}(\omega, \widetilde{x})= \begin{cases}\left\{f_{i}(\omega, \widetilde{x})\right\} & \text { if }(\omega, \widetilde{x}) \in U_{i} ; \\ \operatorname{cl}\left(A_{i}(\omega, \widetilde{x}) a_{i}(\widetilde{x})\right. & \text { if }(\omega, \widetilde{x}) \notin U_{i} .\end{cases}$

Define $G_{i}^{\prime}: L_{X} \rightarrow 2^{L_{X_{i}}}$, by $G_{i}^{\prime}(\widetilde{x})=\left\{y_{i} \in L_{X_{i}}: y_{i}(\omega) \in G_{i}(\omega, \widetilde{x}) \mu\right.$-a.e. $\}$ and $G^{\prime}: L_{X} \rightarrow 2^{L_{X}}$ by $G^{\prime}(\widetilde{x}):=\prod_{i \in I} G_{i}^{\prime}(\widetilde{x})$ for each $\tilde{x} \in L_{X}$. We shall prove that $G^{\prime}$ is an upper semicontinuous correspondence with respect to the weakly topology of $L_{X}$ and has nonempty convex closed values. By applying Fan-Glicksberg's fixed-point Theorem [30] to $G^{\prime}$, we obtain a fixed point, which is the equilibrium point for the abstract economy.

It follows by Theorem III.40 in [31] and the projection theorem that for each $\tilde{x} \in L_{X}$, the correspondence $\tilde{x} \rightarrow \operatorname{cl}\left(A_{i}(\cdot, \tilde{x})\right)_{a_{i}(\tilde{x})}: \Omega \rightarrow 2^{Y}$ has a measurable graph. For each $\tilde{x} \in$ $L_{X}$, the correspondence $G_{i}(\cdot, \tilde{x})$ has a measurable graph. Since $\Phi_{i}(\omega, \cdot)$ has weakly open lower sections for each $\omega \in \Omega$, it follows that $U_{i}^{\omega}$ is weakly open in $L_{X}$. By Lemma 1, for each $\omega \in \Omega, G_{i}(\omega, \cdot): L_{X} \rightarrow 2^{Y}$ is upper semi-continuous in the sense that the set $\left\{\tilde{x} \in L_{X}\right.$ : $\left.G_{i}(\omega, \widetilde{x})\right\} \subset V$ is weakly open in $L_{X}$ for every norm open subset $V$ of $Y$. Moreover, $G_{i}$ is convex and nonempty-valued.

$G_{i}$ is nonempty-valued, and for each $\tilde{x} \in L_{X}, G_{i}(\cdot, \tilde{x})$ has a measurable graph. Hence, according to the Aumann measurable selection theorem for each fixed $\tilde{x} \in L_{X}$, there exists an $\mathcal{F}_{i}$-measurable function $y_{i}: \Omega \rightarrow Y$ such that $y_{i}(\omega) \in G_{i}(\omega, \widetilde{x}) \mu$-a.e. Since for each $(\omega, \widetilde{x}) \in \Omega \times L_{X}, G_{i}(\omega, \widetilde{x})$ is contained in the values of the integrably bounded correspondence $X_{i}(\cdot)$, then $y_{i} \in L_{X_{i}}$, and we conclude that $y_{i} \in G_{i}^{\prime}(\widetilde{x})$ for each $\widetilde{x} \in L_{X}$. Thus, $G_{i}^{\prime}$ is nonempty-valued.

Since for each $\tilde{x} \in L_{X}, G_{i}(\cdot, \widetilde{x})$ has a measurable graph and for each $\omega \in \Omega, G_{i}(\omega, \cdot): L_{X} \rightarrow$ $2^{Y}$ is upper semicontinuous and $G_{i}(\omega, \widetilde{x}) \subset\left(X_{i}(\omega)\right)_{z_{i}}$ for each $(\omega, \widetilde{x}) \in \Omega \times L_{X}$, by u.s.c. lifting theorem (see Appendix), it follows that $G_{i}^{\prime}$ is weakly upper semicontinuous. $G_{i}^{\prime}$ is convex-valued since $G_{i}$ is such.

$G^{\prime}$ is a weakly upper semicontinuous correspondence, and it also has nonempty convex closed values.

The set $L_{X}$ is weakly compact and convex, and then, by Fan-Glicksberg's fixed-point theorem in [30], there exists $\widetilde{x}^{*} \in L_{X}$ such that $\widetilde{x}^{*} \in G^{\prime}\left(\widetilde{x}^{*}\right)$, i.e., for each $i \in I, \widetilde{x}_{i}^{*} \in G_{i}^{\prime}\left(\widetilde{x}^{*}\right)$.

Then, $\widetilde{x}_{i}^{*} \in L_{X_{i}}$ and $\widetilde{x}_{i}^{*}(\omega) \in G_{i}\left(\omega, \widetilde{x}^{*}\right) \mu$-a.e. Since $\widetilde{x}_{i}^{*}(\omega) \notin\left(A_{i}\left(\omega, \widetilde{x}^{*}\right)\right)_{a_{i}\left(\widetilde{x}^{*}\right)} \cap\left(P_{i}\left(\omega, \widetilde{x}^{*}\right)\right)_{p_{i}\left(\widetilde{x}^{*}\right)}$ $\mu$-a.e., it follows that $\left(\omega, \widetilde{x}^{*}\right) \notin U_{i}$ for each $i \in I$ and $\widetilde{x}_{i}^{*} \in \operatorname{cl}\left(A_{i}\left(\omega, \widetilde{x}^{*}\right)\right)_{a_{i}\left(\widetilde{x}^{*}\right)} \mu$-a.e. We also have that $\left(A_{i}\left(\omega, \widetilde{x}^{*}\right)\right)_{a_{i}\left(\widetilde{x}^{*}\right)} \cap\left(P_{i}\left(\omega, \widetilde{x}^{*}\right)\right)_{p_{i}\left(\widetilde{x}^{*}\right)}=\emptyset$.

Example 1 Let $(\Omega, \mathcal{F}, \mu)$ be the measure space, where $\Omega=[0,1], \mathcal{F}=\beta([0,1])$ is the $\sigma$ algebra of the Borel measurable subsets in $[0,1]$ and $\mu$ is the Lebesgue measure.

Let $Y=\mathbb{R}$ and $I=\{1,2, \ldots, n\}$.

For each $i \in I$, let us define the following.

$$
\mathcal{F}_{i}=\mathcal{F}
$$

The random fuzzy constraint function $z_{i}:[0,1] \rightarrow(0,1]$ is defined by

$$
z_{i}(\omega)=\frac{1}{i+2} \quad \text { if } \omega \in[0,1]
$$


The random fuzzy mapping $X_{i}(\cdot):[0,1] \rightarrow \mathcal{F}(\mathbb{R})$ is defined by

$$
X_{i}(\omega)(y)= \begin{cases}0 & \text { if } \omega \in[0,1] \text { and } y \in(-\infty, 0) \cup(1, \infty) \\ \frac{2}{i+5} y+\frac{2}{i+2} & \text { if } \omega \in[0,1] \text { and } y \in[0,1]\end{cases}
$$

Then, the correspondence $X_{i}:[0,1] \rightarrow 2^{\mathbb{R}}$ is defined by

$$
X_{i}(\omega)=\left\{y \in \mathbb{R}: \frac{2}{i+5} y+\frac{2}{i+2}>\frac{1}{i+2} \text { and } y \in[0,1]\right\}=[0,1] \quad \text { for each } \omega \in[0,1] \text {. }
$$

It is a nonempty convex compact valued and integrably bounded correspondence. It is also $\mathcal{F}_{i}$-lower measurable.

Let $L_{X_{i}}=\left\{x_{i} \in S_{\left(X_{i} \cdot(\cdot)\right)_{i}}: x_{i}\right.$ is $\mathcal{F}_{i}$-measurable $\}$ and $L_{X}=\prod_{i \in I} L_{X_{i}}$.

The random fuzzy constraint function $a_{i}: L_{X} \rightarrow(0,1]$ is defined by

$$
a_{i}(\widetilde{x})=\frac{1}{2} \quad \text { for each } \tilde{x} \in L_{X}
$$

For each $\omega \in[0,1]$, the random fuzzy constraint mapping of agent $i, A_{i}(\omega, \cdot): L_{X} \rightarrow \mathcal{F}(\mathbb{R})$ is defined by

$$
A_{i}(\omega, \widetilde{x})(y)= \begin{cases}\frac{5}{10 y+2} & \text { if }(\omega, \widetilde{x}) \in[0,1] \times L_{X} \text { and } y \in(0,1] \\ 0 & \text { if }(\omega, \widetilde{x}) \in[0,1] \times L_{X} \text { and } y \in(-\infty, 0] \cup(1, \infty)\end{cases}
$$

Then, the correspondence $(\omega, \widetilde{x}) \rightarrow\left(A_{i}(\omega, \widetilde{x})\right)_{a_{i}(\widetilde{x})}:[0,1] \times L_{X} \rightarrow 2^{[0,1]}$ is defined by

$$
\begin{aligned}
\left(A_{i}(\omega, \widetilde{x})\right)_{a_{i}(\widetilde{x})} & =\left\{y \in[0,1]: A_{i}(\omega, \widetilde{x})(y)>a_{i}(\widetilde{x})\right\} \\
& =\left\{y \in(0,1]: \frac{5}{10 y+1}>\frac{1}{2}\right\}=\left\{y \in(0,1]: y<\frac{9}{10}\right\}=\left(0, \frac{9}{10}\right) .
\end{aligned}
$$

For each $\omega \in[0,1]$, it has weakly open lower sections in $L_{X}$, and it has a measurable graph.

For each $(\omega, \widetilde{x}) \in[0,1] \times L_{X},\left(A_{i}(\omega, \widetilde{x})\right)_{a_{i}(\widetilde{x})}$ is convex and with nonempty interior in $[0,1]$. For each $\omega \in[0,1]$, the correspondence $\tilde{x} \rightarrow \operatorname{cl}\left(A_{i}(\omega, \widetilde{x})\right)_{a_{i}(\tilde{x})}: L_{X} \rightarrow 2^{[0,1]}$, defined by $\operatorname{cl}\left(A_{i}(\omega, \widetilde{x})\right)_{a_{i}(\tilde{x})}=\left[0, \frac{9}{10}\right]$ for each $\widetilde{x} \in L_{X}$ is upper semicontinuous and nonempty-valued.

The random fuzzy preference mapping $p_{i}: L_{X} \rightarrow(0,1]$ is defined by

$$
p_{i}(\widetilde{x})=\frac{1}{5} \quad \text { for each } \tilde{x} \in L_{X}
$$

Let us define $D_{i}=\prod_{j \neq i} L_{X_{j}} \times\left\{\widetilde{x}_{i}:[0,1] \rightarrow[0,1], \widetilde{x}_{i}(\omega)=k_{\widetilde{x}_{i}} \omega^{i}, \omega \in[0,1], k_{\widetilde{x}_{i}} \in[0,1]\right\} . D_{i}$ is weakly closed in $L_{X}$.

For each $\omega \in[0,1]$, the random fuzzy preference mapping of agent $i, P_{i}(\omega, \cdot): L_{X} \rightarrow \mathcal{F}(\mathbb{R})$ is defined by

$$
P_{i}(\omega, \widetilde{x})(y)= \begin{cases}\frac{5 y+2}{\left.55 x_{i}(\omega)+6\right)} & \text { if }(\omega, \widetilde{x}) \in[0,1] \times D_{i} \text { and } y \in(0,1] \\ 0 & \text { otherwise. }\end{cases}
$$


Then, for each $\omega \in[0,1]$, the correspondence $\widetilde{x} \rightarrow\left(P_{i}(\omega, \widetilde{x})\right)_{p_{i}(\widetilde{x})}: L_{X} \rightarrow 2^{[0,1]}$ is defined by

$$
\begin{aligned}
\left(P_{i}(\omega, \widetilde{x})\right)_{p_{i}(\tilde{x})} & = \begin{cases}\left\{y \in[0,1): P_{i}(\omega, \tilde{x})(y)>p_{i}(\widetilde{x})\right\} & \text { if } \tilde{x} \in D_{i} ; \\
\emptyset & \text { if } \tilde{x} \notin D_{i}\end{cases} \\
& = \begin{cases}\left.\frac{\tilde{x}_{i}(\omega)+4}{5}, 1\right) & \text { if } \tilde{x} \in D_{i} ; \\
\emptyset & \text { if } \tilde{x} \notin D_{i} .\end{cases}
\end{aligned}
$$

For each $\omega \in \Omega$ and for each $y \in Y$, the set $\left(\left(P_{i}(\omega, \widetilde{x})\right)_{p_{i}(\widetilde{x})}\right)^{-1}(\omega, y)=\left\{\tilde{x} \in D_{i}: 0 \leq \widetilde{x}_{i}(\omega)<\right.$ $5 y-4\}$ is weakly open in $D_{i}$, then it is weakly open in $L_{X}$. Therefore, the correspondence $(\omega, \widetilde{x}) \rightarrow\left(P_{i}(\omega, \widetilde{x})\right)_{p_{i}(\widetilde{x})}$ has weakly open lower sections. It also has open convex values and a measurable graph.

For each $i \in I, \widetilde{x}_{i}(\omega) \notin\left(P_{i}(\omega, \widetilde{x})\right)_{p_{i}(\widetilde{x}}$, for each $\omega \in[0,1]$ and $\widetilde{x} \in L_{X}$.

All the assumptions of Theorem 1 are fulfilled, then an equilibrium exists.

For example, $\widetilde{x}^{*} \in L_{X}$ such that for each $i \in I, \widetilde{x}_{i}^{*}(\omega)=\frac{3}{4} \omega^{i}, \omega \in[0,1]$ is an equilibrium for the abstract fuzzy economy, that is, for each $i \in I$ and $\mu$-a.e.:

$$
\widetilde{x_{i}^{*}}(\omega) \in \operatorname{cl}\left(A_{i}(\omega, \widetilde{x})\right)_{a_{i}(\tilde{x})} \quad \text { and } \quad\left(A_{i}(\omega, \widetilde{x})\right)_{a_{i}(\tilde{x})} \cap\left(P_{i}(\omega, \widetilde{x})\right)_{p_{i}(\widetilde{x})}=\emptyset .
$$

\section{Random quasi-variational inequalities}

In this section, we are establishing new random quasi-variational inequalities with random fuzzy mappings and random fixed point theorems. The proofs rely on the theorem of Bayesian fuzzy equilibrium existence for the Bayesian abstract fuzzy economy.

This is our first theorem.

Theorem 2 Let I be a countable or uncountable set. Let $(\Omega, \mathcal{F}, \mu)$ be a complete finite separable measure space, and let $Y$ be a separable Banach space. Suppose that the following conditions are satisfied.

For each $i \in I$ :

(A.1) (a) $X_{i}: \Omega \rightarrow \mathcal{F}(Y)$ is such that $\omega \rightarrow\left(X_{i}(\omega)\right)_{z_{i}}: \Omega \rightarrow 2^{Y}$ is a nonempty convex weakly compact-valued and integrably bounded correspondence;

(b) $X_{i}: \Omega \rightarrow \mathcal{F}(Y)$ is such that $\omega \rightarrow\left(X_{i}(\omega)\right)_{z_{i}}: \Omega \rightarrow 2^{Y}$ is $\mathcal{F}_{i}$-lower measurable;

(A.2) (a) For each $(\omega, \widetilde{x}) \in \Omega \times L_{X},\left(A_{i}(\omega, \widetilde{x})\right)_{a_{i}(\widetilde{x})}$ is convex and has a nonempty interior in the relative norm topology of $\left(X_{i}(\omega)\right)_{z_{i}}$;

(b) the correspondence $(\omega, \widetilde{x}) \rightarrow\left(A_{i}(\omega, \widetilde{x})\right)_{a_{i}(\tilde{x})}: \Omega \times L_{X} \rightarrow 2^{Y}$ has a measurable graph, i.e., $\left\{(\omega, \widetilde{x}, y) \in \Omega \times L_{X} \times Y: y \in\left(A_{i}(\omega, \widetilde{x})\right)_{a_{i}(\tilde{x})}\right\} \in \mathcal{F} \otimes \beta_{w}\left(L_{X}\right) \otimes \beta(Y)$, where $\beta_{w}\left(L_{X}\right)$ is the Borel $\sigma$-algebra for the weak topology on $L_{X}$ and $\beta(Y)$ is the Borel $\sigma$-algebra for the norm topology on $Y$;

(c) the correspondence $(\omega, \widetilde{x}) \rightarrow\left(A_{i}(\omega, \widetilde{x})\right)_{a_{i}(\widetilde{x})}$ has weakly open lower sections, i.e., for each $\omega \in \Omega$ and for each $y \in Y$, the set $\left(\left(A_{i}(\omega, \widetilde{x})\right)_{a_{i}(\widetilde{x})}\right)^{-1}(\omega, y)=\left\{\widetilde{x} \in L_{X}: y \in\left(A_{i}(\omega, \widetilde{x})\right)_{a_{i}(\widetilde{x})}\right\}$ is weakly open in $L_{X} ;$

(d) For each $\omega \in \Omega, \widetilde{x} \rightarrow \operatorname{cl}\left(A_{i}(\omega, \widetilde{x})\right)_{a_{i}(\tilde{x})}: L_{X} \rightarrow 2^{Y}$ is upper semicontinuous in the sense that the set $\left\{\tilde{x} \in L_{X}: \operatorname{cl}\left(A_{i}(\omega, \tilde{x})\right)_{a_{i}(\tilde{x})} \subset V\right\}$ is weakly open in $L_{X}$ for every norm open subset $V$ of $Y$;

(A.3) $\psi_{i}: \Omega \times L_{X} \times Y \rightarrow \mathbb{R} \cup\{-\infty,+\infty\}$ is such that: 
(a) $\tilde{x} \rightarrow \psi_{i}(\omega, \tilde{x}, y)$ is lower semicontinuous on $L_{X}$ for each fixed $(\omega, y) \in \Omega \times Y$;

(b) $\widetilde{x_{i}}(\omega) \notin\left\{y \in Y: \psi_{i}(\omega, \tilde{x}, y)>0\right\}$ for each fixed $(\omega, \widetilde{x}) \in \Omega \times L_{X}$;

(c) for each $(\omega, \tilde{x}) \in \Omega \times L_{X}, \psi_{i}(\omega, \tilde{x}, \cdot)$ is quasiconcave;

(d) for each $\omega \in \Omega,\left\{\tilde{x} \in L_{X}: \alpha_{i}(\omega, \tilde{x})>0\right\}$ is weakly open in $L_{X}$, where $\alpha_{i}: \Omega \times L_{X} \rightarrow R$ is defined by $\alpha_{i}(\omega, \widetilde{x})=\sup _{y \in\left(A_{i}(\omega, \tilde{x})\right)_{a_{i}(\tilde{x})}} \psi_{i}(\omega, \tilde{x}, y)$ for each $(\omega, \widetilde{x}) \in \Omega \times L_{X}$;

(e) $\left\{(\omega, \widetilde{x}): \alpha_{i}(\omega, \widetilde{x})>0\right\} \in \mathcal{F}_{i} \otimes B\left(L_{X}\right)$.

Then, there exists $\widetilde{x}^{*} \in L_{X}$ such that for every $i \in I$ and $\mu$-a.e.:

(i) $\widetilde{x}_{i}^{*}(\omega) \in \operatorname{cl}\left(A_{i}\left(\omega, \widetilde{x}^{*}\right)\right)_{a_{i}\left(\widetilde{x}^{*}\right)}$;

(ii) $\sup _{y \in\left(A_{i}\left(\omega, \widetilde{x}^{*}\right)\right)_{a_{i}\left(\tilde{x}^{*}\right)}} \psi_{i}\left(\omega, \widetilde{x}^{*}, y\right) \leq 0$.

Proof For every $i \in I$, let $P_{i}: \Omega \times S_{X}^{1} \rightarrow \mathcal{F}(Y)$, and let $p_{i}: L_{X} \rightarrow(0,1]$ such that $\left(P_{i}(\omega, \widetilde{x})\right)_{p_{i}(\widetilde{x})}=\left\{y \in Y: \psi_{i}(\omega, \widetilde{x}, y)>0\right\}$ for each $(\omega, \widetilde{x}) \in \Omega \times L_{X}$.

We shall show that the abstract economy $G=\left\{(\Omega, \mathcal{F}, \mu),\left(X_{i}, \mathcal{F}_{i}, A_{i}, P_{i} a_{i}, p_{i}, z_{z}\right)_{i \in I}\right\}$ satisfies all hypotheses of Theorem 1.

Suppose $\omega \in \Omega$.

According to (A.3)(a), we have that $\widetilde{x} \rightarrow\left(P_{i}(\omega, \widetilde{x})\right)_{p_{i}(\tilde{x}}: \Omega \rightarrow 2^{Y}$ has open lower sections, nonempty compact values and according to (A.3)(b), $\widetilde{x_{i}}(\omega) \notin\left(P_{i}(\omega, \widetilde{x})\right)_{p_{i}(\tilde{x})}$ for each $\widetilde{x} \in L_{X}$. Assumption (A.3)(c) implies that $\widetilde{x} \rightarrow\left(P_{i}(\omega, \widetilde{x})\right)_{p_{i}(\widetilde{x})}: \Omega \rightarrow 2^{Y}$ has convex values.

By the definition of $\alpha_{i}$, we note that $\left\{\tilde{x} \in L_{X}:\left(A_{i}(\omega, \widetilde{x})\right)_{a_{i}(\tilde{x})} \cap\left(P_{i}(\omega, \widetilde{x})\right)_{p_{i}(\tilde{x})} \neq \emptyset\right\}=\{\tilde{x} \in$ $\left.L_{X}: \alpha_{i}(\omega, \widetilde{x})>0\right\}$ so that $\left\{\widetilde{x} \in L_{X}:\left(A_{i}(\omega, \widetilde{x})\right)_{a_{i}(\widetilde{x})} \cap\left(P_{i}(\omega, \widetilde{x})\right)_{p_{i}(\widetilde{x})} \neq \emptyset\right\}$ is weakly open in $L_{X}$ by (A.3)(d).

According to (A.2)(b) and (A.3)(e), it follows that the correspondences $(\omega, \widetilde{x}) \rightarrow$ $\left(A_{i}(\omega, \widetilde{x})\right)_{a_{i}(\tilde{x})}: \Omega \times L_{X} \rightarrow 2^{Y}$ and $(\omega, \widetilde{x}) \rightarrow\left(P_{i}(\omega, \widetilde{x})\right)_{p_{i}(\widetilde{x})}: \Omega \times L_{X} \rightarrow 2^{Y}$ have measurable graphs.

Thus, the Bayesian abstract fuzzy economy $G=\left\{(\Omega, \mathcal{F}, \mu),\left(X_{i}, \mathcal{F}_{i}, A_{i}, P_{i}, a_{i}, b_{i}, z_{i}\right)_{i \in I}\right\}$ satisfies all hypotheses of Theorem 1 . Therefore, there exists $\widetilde{x}^{*} \in L_{X}$ such that for every $i \in I$ :

$$
\widetilde{x}_{i}^{*}(\omega) \in \operatorname{cl}\left(A_{i}\left(\omega, \widetilde{x}^{*}\right)\right)_{a_{i}\left(\tilde{x}^{*}\right)} \mu \text {-a.e. } \quad \text { and } \quad\left(A_{i}\left(\omega, \widetilde{x}^{*}\right)\right)_{a_{i}\left(\widetilde{x}^{*}\right)} \cap\left(P_{i}\left(\omega, \widetilde{x}^{*}\right)\right)_{p_{i}\left(\tilde{x}^{*}\right)}=\phi \mu \text {-a.e.; }
$$

that is, there exists $\widetilde{x}^{*} \in L_{X}$ such that for every $i \in I$ and $\mu$-a.e.:

(i) $\widetilde{x}_{i}^{*}(\omega) \in \operatorname{cl}\left(A_{i}\left(\omega, \widetilde{x}^{*}\right)\right)_{a_{i}\left(\widetilde{x}^{*}\right)}$;

(ii) $\sup _{y \in\left(A_{i}\left(\omega, \widetilde{x}^{*}\right)\right)_{a_{i}\left(\tilde{x}^{*}\right)}} \psi_{i}\left(\omega, \widetilde{x}^{*}, y\right) \leq 0$.

Example 2 Let $\Omega=[0,1], \mathcal{F}=\beta([0,1], \mu), Y=\mathbb{R}, I=\{1,2, \ldots, n\}$, and for each $i \in I$, let $\mathcal{F}_{i}$, $X_{i}, a_{i}$ and $D_{i}$ be defined as in Example 1 .

Let us define $\psi_{i}:[0,1] \times L_{X} \times \mathbb{R} \rightarrow \mathbb{R}$ as follows: if $\widetilde{x_{i}}(\omega) \in[0,1)$, then,

$$
\psi_{i}(\omega, \tilde{x}, y)= \begin{cases}1 & \text { if } y \in\left(\frac{\tilde{x}_{i}(\omega)+4}{5}, 1\right) \text { and }(\omega, \widetilde{x}) \in[0,1] \times D_{i} \\ 0 & \text { otherwise }\end{cases}
$$

and if $\widetilde{x_{i}}(\omega)=1, \psi_{i}(\omega, \widetilde{x}, y)=0$ for each $(\omega, \widetilde{x}, y) \in[0,1] \times L_{X} \times \mathbb{R}$.

For each $i \in I$, let $P_{i}: \Omega \times L_{X} \rightarrow \mathcal{F}(Y)$, and let $p_{i}: L_{X} \rightarrow(0,1]$ as in Example 1, and then, $\left(P_{i}(\omega, \widetilde{x})\right)_{p_{i}(\widetilde{x})}=\left\{y \in Y: \psi_{i}(\omega, \widetilde{x}, y)>0\right\}= \begin{cases}\left(\frac{\widetilde{x}_{i}(\omega)+4}{5}, 1\right) & \text { if } \tilde{x} \in D_{i} ; \\ \emptyset & \text { if } \tilde{x} \notin D_{i}\end{cases}$

From the Example 1, we have that for every $i \in I$ and for each $\omega \in[0,1]$, the correspondence $\widetilde{x} \rightarrow\left(P_{i}(\omega, \widetilde{x})\right)_{p_{i}(\widetilde{x})}: L_{X} \rightarrow 2^{[0,1]}$ has weakly open lower sections, open convex values 
and $\tilde{x}_{i}(\omega) \notin\left(P_{i}(\omega, \tilde{x})\right)_{p_{i}(\tilde{x})}$.

$$
\begin{aligned}
\alpha_{i}(\omega, \tilde{x}) & =\sup _{y \in\left(A_{i}(\omega, \widetilde{x})\right)_{a_{i}(\tilde{x})}} \psi_{i}(\omega, \tilde{x}, y) \\
& =\sup _{y \in\left(0, \frac{9}{10}\right)} \psi_{i}(\omega, \tilde{x}, y)= \begin{cases}1 & \text { if } \tilde{x}_{i}(\omega) \in\left[0, \frac{1}{2}\right) ; \\
0 & \text { if } \tilde{x}_{i}(\omega) \in\left[\frac{1}{2}, 1\right] .\end{cases}
\end{aligned}
$$

By the definition of $\alpha_{i}$, we note that for each $\omega \in \Omega, N_{i}(\omega)=\left\{\tilde{x} \in L_{X}: \alpha_{i}(\omega, \widetilde{x})>0\right\}=\{\tilde{x} \in$ $\left.D_{i}: \widetilde{x}_{i}(\omega) \in\left[0, \frac{1}{2}\right)\right\}$ is weakly open in $L_{X}$ and $N_{i} \in \mathcal{F}_{i} \otimes B\left(L_{X}\right)$.

In Example 1, we proved that the correspondence $(\omega, \widetilde{x}) \rightarrow\left(A_{i}(\omega, \widetilde{x})\right)_{a_{i}(\widetilde{x})}: \Omega \times L_{X} \rightarrow 2^{[0,1]}$ has a measurable graph.

Therefore, there exists $\widetilde{x}^{*} \in L_{X}$ such that, for each $i \in I$ and $\mu$-a.e.:

(i) $\widetilde{x}_{i}^{*}(\omega) \in \operatorname{cl}\left(A_{i}\left(\omega, \widetilde{x}^{*}\right)\right)_{a_{i}\left(\tilde{x}^{*}\right)}$;

(ii) $\sup _{y \in\left(A_{i}\left(\omega, \widetilde{x}^{*}\right)\right)_{a_{i}\left(\tilde{x}^{*}\right)}} \psi_{i}\left(\omega, \widetilde{x}^{*}, y\right) \leq 0$.

For instance, $\widetilde{x}^{*}$ is a solution for the variational inequality, where $\widetilde{x}^{*}$ is defined by $\widetilde{x}_{i}^{*}(\omega)=$ $\frac{3}{4} \omega^{i}$ for each $i \in\{1,2, \ldots, n\}$ and $\omega \in \Omega$.

If $|\mathrm{I}|=1$, we obtain the following corollary of Theorem 2.

Corollary 1 Let $(\Omega, \mathcal{F}, \mu)$ be a complete finite separable measure space, and let $Y$ be a separable Banach space. Suppose that the following conditions are satisfied:

(A.1) (a) $X: \Omega \rightarrow \mathcal{F}(Y)$ is such that $\omega \rightarrow(X(\omega))_{z}: \Omega \rightarrow 2^{Y}$ is a nonempty convex weakly compact-valued and integrably bounded correspondence;

(b) $X: \Omega \rightarrow \mathcal{F}(Y)$ is such that $\omega \rightarrow(X(\omega))_{z}: \Omega \rightarrow 2^{Y}$ is $\mathcal{F}$-lower measurable;

(A.2) (a) For each $(\omega, \widetilde{x}) \in \Omega \times L_{X},(A(\omega, \widetilde{x}))_{a(\widetilde{x})}$ is convex and has a nonempty interior in the relative norm topology of $(X(\omega))_{z}$;

(b) the correspondence $(\omega, \widetilde{x}) \rightarrow(A(\omega, \widetilde{x}))_{a_{i}(\tilde{x})}: \Omega \times L_{X} \rightarrow 2^{Y}$ has a measurable graph, i.e., $\left\{(\omega, \widetilde{x}, y) \in \Omega \times L_{X} \times Y: y \in(A(\omega, \widetilde{x}))_{a(\tilde{x})}\right\} \in \mathcal{F} \otimes \beta_{w}\left(L_{X}\right) \otimes \beta(Y)$ where $\beta_{w}\left(L_{X}\right)$ is the Borel $\sigma$-algebra for the weak topology on $L_{X}$ and $\beta(Y)$ is the Borel $\sigma$-algebra for the norm topology on $Y$;

(c) the correspondence $(\omega, \widetilde{x}) \rightarrow(A(\omega, \widetilde{x}))_{a(\tilde{x})}$ has weakly open lower sections, i.e., for each $\omega \in \Omega$ and for each $y \in Y$, the set $\left((A(\omega, \widetilde{x}))_{a(\widetilde{x}}\right)^{-1}(\omega, y)=\left\{\widetilde{x} \in L_{X}: y \in(A(\omega, \widetilde{x}))_{a(\widetilde{x})}\right\}$ is weakly open in $L_{X} ;$

(d) For each $\omega \in \Omega, \widetilde{x} \rightarrow \operatorname{cl}(A(\omega, \widetilde{x}))_{a(\tilde{x})}: L_{X} \rightarrow 2^{Y}$ is upper semicontinuous in the sense that the set $\left\{\tilde{x} \in L_{X}: \operatorname{cl}(A(\omega, \widetilde{x}))_{a(\widetilde{x})} \subset V\right\}$ is weakly open in $L_{X}$ for every norm open subset $V$ of $Y$;

(A.3) $\psi: \Omega \times L_{X} \times Y \rightarrow R \cup\{-\infty,+\infty\}$ is such that:

(a) $\tilde{x} \rightarrow \psi(\omega, \tilde{x}, y)$ is lower semicontinuous on $L_{X}$ for each fixed $(\omega, y) \in \Omega \times Y$;

(b) $\widetilde{x}(\omega) \notin\{y \in Y: \psi(\omega, \widetilde{x}, y)>0\}$ for each fixed $(\omega, \widetilde{x}) \in \Omega \times L_{X}$;

(c) for each $(\omega, \tilde{x}) \in \Omega \times L_{X}, \psi(\omega, \tilde{x}, \cdot)$ is quasiconcave;

(d) for each $\omega \in \Omega,\left\{\tilde{x} \in L_{X}: \alpha(\omega, \tilde{x})>0\right\}$ is weakly open in $L_{X}$, where $\alpha: \Omega \times L_{X} \rightarrow R$ is defined by $\alpha(\omega, \widetilde{x})=\sup _{y \in(A(\omega, \tilde{x}))_{a(x)}} \psi(\omega, \tilde{x}, y)$ for each $(\omega, \widetilde{x}) \in \Omega \times L_{X}$

(e) $\{(\omega, \widetilde{x}): \alpha(\omega, \widetilde{x})>0\} \in \mathcal{F} \otimes B\left(L_{X}\right)$;

Then, there exists $\widetilde{x}^{*} \in L_{X}$ such that $\mu$-a.e.:

(i) $\widetilde{x}^{*}(\omega) \in \operatorname{cl}\left(A\left(\omega, \widetilde{x}^{*}\right)\right)_{a\left(\widetilde{x}^{*}\right)}$;

(ii) $\sup _{y \in\left(A\left(\omega, \widetilde{x}^{*}\right)\right)_{a\left(\tilde{x}^{*}\right)}} \psi\left(\omega, \tilde{x}^{*}, y\right) \leq 0$. 
As a consequence of Theorem 2, we prove the following Tan and Yuan-type [22] random quasi-variational inequality with random fuzzy mappings.

Theorem 3 Let $(\Omega, \mathcal{F}, \mu)$ be a complete finite separable measure space, and let $Y$ be a separable Banach space. Suppose that the following conditions are satisfied:

For each $i \in I$ :

(A.1) (a) $X_{i}: \Omega \rightarrow \mathcal{F}(Y)$ is such that $\omega \rightarrow\left(X_{i}(\omega)\right)_{z_{i}}: \Omega \rightarrow 2^{Y}$ is a nonempty convex weakly compact-valued and integrably bounded correspondence;

(b) $X_{i}: \Omega \rightarrow \mathcal{F}(Y)$ is such that $\omega \rightarrow\left(X_{i}(\omega)\right)_{z_{i}}: \Omega \rightarrow 2^{Y}$ is $\mathcal{F}_{i}$-lower measurable;

A.2) (a) For each $(\omega, \tilde{x}) \in \Omega \times L_{X},\left(A_{i}(\omega, \widetilde{x})\right)_{a_{i}(\tilde{x})}$ is convex and has a nonempty interior in the relative norm topology of $\left(X_{i}(\omega)\right)_{z_{i}}$;

(b) the correspondence $(\omega, \tilde{x}) \rightarrow\left(A_{i}(\omega, \widetilde{x})\right)_{a_{i}(\tilde{x})}: \Omega \times L_{X} \rightarrow 2^{Y}$ has a measurable graph, i.e., $\left\{(\omega, \tilde{x}, y) \in \Omega \times L_{X} \times Y: y \in\left(A_{i}(\omega, \tilde{x})\right)_{a_{i}(\tilde{x})}\right\} \in \mathcal{F} \otimes \beta_{w}\left(L_{X}\right) \otimes \beta(Y)$ where $\beta_{w}\left(L_{X}\right)$ is the Borel $\sigma$-algebra for the weak topology on $L_{X}$ and $\beta(Y)$ is the Borel $\sigma$-algebra for the norm topology on $Y$;

(c) the correspondence $(\omega, \tilde{x}) \rightarrow\left(A_{i}(\omega, \tilde{x})\right)_{a_{i}(\widetilde{x})}$ has weakly open lower sections, i.e., for each $\omega \in \Omega$ and for each $y \in Y$, the set $\left(\left(A_{i}(\omega, \tilde{x})\right)_{a_{i}(\tilde{x})}\right)^{-1}(\omega, y)=\left\{\tilde{x} \in L_{X}: y \in\left(A_{i}(\omega, \tilde{x})\right)_{a_{i}(\widetilde{x})}\right\}$ is weakly open in $L_{X} ;$

(d) For each $\omega \in \Omega, \tilde{x} \rightarrow \operatorname{cl}\left(A_{i}(\omega, \tilde{x})\right)_{a_{i}(\tilde{x})}: L_{X} \rightarrow 2^{Y}$ is upper semicontinuous in the sense that the set $\left\{\tilde{x} \in L_{X}: \operatorname{cl}\left(A_{i}(\omega, \tilde{x})\right)_{a_{i}(\tilde{x})} \subset V\right\}$ is weakly open in $L_{X}$ for every norm open subset $V$ of $Y$;

(A.3) $G_{i}: \Omega \times Y \rightarrow \mathcal{F}\left(Y^{\prime}\right)$ and $g_{i}: Y \rightarrow(0,1]$ are such that

(a) for each $\omega \in \Omega, y \rightarrow\left(G_{i}(\omega, y)\right)_{g_{i}(y)}: Y \rightarrow 2^{Y^{\prime}}$ is monotone (that is $\operatorname{Re}\langle u-v, y-x\rangle \geq 0$ for all $u \in\left(G_{i}(\omega, y)\right)_{g_{i}(y)}$ and $v \in\left(G_{i}(\omega, x)\right)_{g_{i}(x)}$ and $\left.x, y \in Y\right)$ with nonempty values;

(b) for each $\omega \in \Omega, y \rightarrow\left(G_{i}(\omega, y)\right)_{g_{i}(y)}: L \cap Y \rightarrow 2^{Y^{\prime}}$ is lower semicontinuous from the relative topology of $Y$ into the weak ${ }^{*}$-topology $\sigma\left(Y^{\prime}, Y\right)$ of $Y^{\prime}$ for each one-dimensional flat $L \subset Y$;

(A.4) (a) for each fixed $\omega \in \Omega$, the set $\left\{\tilde{x} \in S_{X}^{1}: \sup _{y \in\left(A_{i}(\omega, \tilde{x})\right)_{a_{i}(\tilde{x})}}\left[\sup _{u \in\left(G_{i}(\omega, y)\right)_{g_{i}(y)}} \operatorname{Re}\langle u, \tilde{x}-y\rangle\right]>0\right\}$ is weakly open in $L_{X}$;

(b) $\left\{(\omega, \widetilde{x}): \sup _{u \in\left(G_{i}(\omega, y)\right)_{g_{i}(y)}} \operatorname{Re}\left\langle u, \widetilde{x}_{i}(\omega)-y\right\rangle>0\right\} \in \mathcal{F} \otimes B\left(L_{X}\right)$.

Then, there exists $\tilde{x}^{*} \in L_{X}$ such that for every $i \in I$ and $\mu$-a.e.:

(i) $\tilde{x}_{i}^{*}(\omega) \in \operatorname{cl}\left(A_{i}\left(\omega, \tilde{x}^{*}\right)\right)_{a_{i}\left(\tilde{x}^{*}\right)}$;

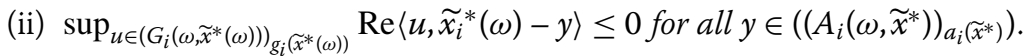

Proof Let us define $\psi_{i}: \Omega \times L_{X} \times Y \rightarrow R \cup\{-\infty,+\infty\}$ by

$$
\psi_{i}(\omega, \tilde{x}, y)=\sup _{u \in\left(G_{i}(\omega, y)\right)_{g_{i}(y)}} \operatorname{Re}\left\langle u, \widetilde{x_{i}}(\omega)-y\right\rangle \quad \text { for each }(\omega, \tilde{x}, y) \in \Omega \times L_{X} \times Y
$$

We have that $\tilde{x} \rightarrow \psi_{i}(\omega, \tilde{x}, y)$ is lower semicontinuous on $L_{X}$ for each fixed $(\omega, y) \in \Omega \times Y$ and $\tilde{x}_{i}(\omega) \notin\left\{y \in Y: \psi_{i}(\omega, \tilde{x}, y)>0\right\}$ for each fixed $(\omega, \tilde{x}) \in \Omega \times L_{X}$.

We also know that for each $(\omega, \tilde{x}) \in \Omega \times L_{X}, \psi_{i}(\omega, \widetilde{x}, \cdot)$ is concave. This fact is a consequence of assumption (A.3)(a).

All the hypotheses of Theorem 2 are satisfied. According to Theorem 2, there exists $\tilde{x}^{*} \in L_{X}$ such that $\tilde{x}_{i}^{*}(\omega) \in \operatorname{cl}\left(A_{i}\left(\omega, \tilde{x}^{*}\right)\right)_{i}\left(\tilde{x}^{*}\right)$ for every $i \in I$ and

(1) $\sup _{y \in\left(A_{i}\left(\omega, \widetilde{x}^{*}\right)\right)_{a_{i}\left(\widetilde{x}^{*}\right)}} \sup _{u \in\left(G_{i}(\omega, y)\right)_{g_{i}(y)}}\left[\operatorname{Re}\left\langle u, \tilde{x}_{i}^{*}(\omega)-y\right\rangle\right] \leq 0$ for every $i \in I$. 
Finally, we will prove that $\sup _{y \in\left(A_{i}\left(\omega, \widetilde{x}^{*}\right)\right)_{a_{i}\left(\widetilde{x}^{*}\right)}} \sup _{u \in\left(G_{i}\left(\omega, \widetilde{x}^{*}(\omega)\right)\right)_{i}\left(\tilde{x}^{*}(\omega)\right)}\left[\operatorname{Re}\left\langle u,{\widetilde{x_{i}}}^{*}(\omega)-y\right\rangle\right] \leq 0$ for every $i \in I$.

In order to do that, let us consider $i \in I$ and the fixed point $\omega \in \Omega$.

Let $y \in\left(A_{i}\left(\omega, \widetilde{x}^{*}\right)\right)_{a_{i}\left(\tilde{x}^{*}\right)}, \lambda \in[0,1]$ and $z_{\lambda}^{i}(\omega):=\lambda y+(1-\lambda) \widetilde{x}_{i}^{*}(\omega)$. According to assumption (A.2)(a), $z_{\lambda}^{i}(\omega) \in A_{i}\left(\omega, \widetilde{x}^{*}\right)$.

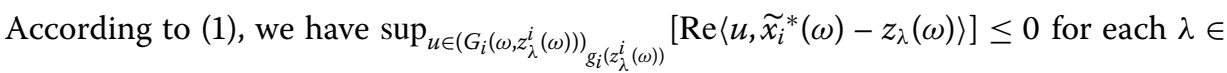
$[0,1]$.

Therefore, for each $\lambda \in[0,1]$, we have that

$$
\begin{aligned}
& t\left\{\sup _{u \in\left(G_{i}\left(\omega, z_{\lambda}^{i}(\omega)\right)\right)_{g_{i}\left(z_{\lambda}^{i}(\omega)\right)}}\left[\operatorname{Re}\left(u,{\widetilde{x_{i}}}^{*}(\omega)-y\right\rangle\right]\right\} \\
& \left.\quad=\sup _{u \in\left(G_{i}\left(\omega, z_{\lambda}^{i}(\omega)\right)\right)_{g_{i}\left(z_{\lambda}^{i}(\omega)\right)}} t\left[\operatorname{Re}\left\langle u, \widetilde{x}_{i}^{*}\left(\omega_{i}\right)-y\right)\right\rangle\right] \\
& \quad=\sup _{u \in\left(G_{i}\left(\omega, z_{\lambda}^{i}(\omega)\right)\right) g_{g_{i}}\left(z_{\lambda}^{i}(\omega)\right)}\left[\operatorname{Re}\left\langle u,{\widetilde{x_{i}}}^{*}(\omega)-z_{\lambda}^{i}(\omega)\right\rangle\right] \leq 0 .
\end{aligned}
$$

It follows that for each $\lambda \in[0,1]$,

(2) $\sup _{u \in\left(G_{i}\left(\omega, z_{\lambda}^{i}(\omega)\right)\right)_{g_{i}\left(z_{\lambda}^{i}(\omega)\right)}}\left[\operatorname{Re}\left\langle u, \widetilde{x}_{i}^{*}(\omega)-y\right\rangle\right] \leq 0$.

Now, we are using the lower semicontinuity of $y \rightarrow\left(G_{i}(\omega, y)\right)_{g_{i}(y)}: L \cap Y \rightarrow 2^{Y^{\prime}}$ in order to show the conclusion. For each $z_{0} \in\left(G_{i}\left(\omega, \widetilde{x}_{i}^{*}(\omega)\right)\right)_{g_{i}\left(\widetilde{x}_{i}{ }^{*}(\omega)\right)}$ and $e>0$ let us consider $U_{z_{0}}^{i}$, the neighborhood of $z_{0}$ in the topology $\sigma\left(Y^{\prime}, Y\right)$, defined by $U_{z_{0}}^{i}:=\left\{z \in Y^{\prime}: \mid \operatorname{Re}\left\langle z_{0}-z, \widetilde{x}_{i}{ }^{*}(\omega)-\right.\right.$ $y\rangle \mid<e\}$. As $y \rightarrow\left(G_{i}(\omega, y)\right)_{g_{i}(y)}: L \cap Y \rightarrow 2^{Y^{\prime}}$ is lower semicontinuous, where $L=\left\{z_{\lambda}^{i}(\omega)\right.$ : $\lambda \in[0,1]\}$ and $U_{z_{0}}^{i} \cap\left(G_{i}\left(\omega, \widetilde{x}_{i}^{*}(\omega)\right)\right)_{g_{i}\left(\widetilde{x}_{i}^{*}(\omega)\right)} \neq \emptyset$, there exists a nonempty neighborhood $N\left(\widetilde{x}_{i}{ }^{*}(\omega)\right)$ of $\widetilde{x}_{i}{ }^{*}(\omega)$ in $L$ such that for each $z \in N\left(\widetilde{x}_{i}^{*}(\omega)\right)$, we have that $U_{z_{0}}^{i} \cap\left(G_{i}(\omega, z)\right)_{g_{i}(z)} \neq$ $\emptyset$. Then there exists $\delta \in(0,1], t \in(0, \delta)$ and $u \in\left(G_{i}\left(\omega, z_{\lambda}^{i}(\omega)\right)\right)_{g_{i}\left(z_{\lambda}^{i}(\omega)\right)} \cap U_{z_{0}}^{i} \neq \emptyset$ such that $\operatorname{Re}\left\langle z_{0}-u, \widetilde{x}_{i}{ }^{*}(\omega)-y\right\rangle<e$. Therefore, $\operatorname{Re}\left\langle z_{0}, \widetilde{x}_{i}^{*}(\omega)-y\right\rangle<\operatorname{Re}\left\langle u_{i}, \widetilde{x}_{i}{ }^{*}(\omega)-y\right\rangle+e$.

It follows that

$$
\operatorname{Re}\left|z_{0},{\widetilde{x_{i}}}^{*}(\omega)-y\right\rangle<\operatorname{Re}\left\langle u, \widetilde{x}_{i}^{*}(\omega)-y\right\rangle+e<e .
$$

The last inequality comes from (2). Since $e>0$ and $z_{0} \in\left(G_{i}\left(\omega, \widetilde{x}_{i}^{*}(\omega)\right)\right)_{g_{i}\left(\widetilde{x}_{i}^{*}(\omega)\right)}$ have been chosen arbitrarily, the next relation holds:

$$
\operatorname{Re}\left|z_{0},{\widetilde{x_{i}}}^{*}(\omega)-y\right\rangle<0
$$

Hence, for each $i \in I$, we have that $\sup _{u \in\left(G_{i}\left(\omega, \widetilde{x}^{*}(\omega)\right)\right)_{g_{i}\left(\tilde{x}^{*}(\omega)\right)}}\left[\operatorname{Re}\left\langle z_{0}, \widetilde{x}_{i}^{*}(\omega)-y\right\rangle\right] \leq 0$ for every $y \in \operatorname{cl}\left(A_{i}\left(\omega, \widetilde{x}^{*}\right)\right)_{a_{i}\left(\tilde{x}^{*}\right)}$.

Example 3 Let $(\Omega, \mathcal{F}, \mu)$ be the measure space, where $\Omega=[0,1], \mathcal{F}=\mathcal{\beta}([0,1])$ is the $\sigma$ algebra of the Borel measurable subsets in $[0,1]$, and $\mu$ is the Lebesgue measure.

Let $Y=\mathbb{R}$ and $I=\{1,2, \ldots, n\}$.

For each $i \in I$, let us define the following

$$
\mathcal{F}_{i}=\mathcal{F}
$$


The correspondence $X_{i}$ is as in Example 1, that is, $\left(X_{i}(\omega)\right)_{z_{i}}=[0,1]$ for each $\omega \in[0,1]$.

For each $\omega \in[0,1],\left(X_{i}(\omega)\right)_{z_{i}}$ is a nonempty convex weakly compactly valued and integrably bounded correspondence. It is also $\mathcal{F}_{i}$-lower measurable.

Let $L_{X_{i}}=\left\{x_{i} \in S_{\left(X_{i}(\cdot)\right)_{z_{i}}}: x_{i}\right.$ is $\mathcal{F}_{i}$-measurable $\}$, and let $L_{X}=\prod_{i \in I} L_{X_{i}}$.

Let us define $D_{i}=\prod_{j \neq i} L_{X_{j}} \times\left\{\widetilde{x}_{i}:[0,1] \rightarrow[0,1], \widetilde{x}_{i}(\omega)=k_{\widetilde{x}_{i}}, \omega \in[0,1], k_{\widetilde{x}_{i}} \in[0,1]\right\} . D_{i}$ is weakly closed in $L_{X}$.

The random fuzzy constraint function $a_{i}: L_{X} \rightarrow(0,1]$ is defined by

$$
a_{i}(\widetilde{x})=\frac{1}{2} \quad \text { for each } \tilde{x} \in L_{X} .
$$

For each $\omega \in[0,1]$, the random fuzzy constraint mapping of agent $i, A_{i}(\omega, \cdot): L_{X} \rightarrow \mathcal{F}(\mathbb{R})$ is defined by

$$
A_{i}(\omega, \widetilde{x})(y)= \begin{cases}\frac{19}{20(y+1)} & \text { if }(\omega, \widetilde{x}) \in[0,1] \times D_{i} \text { and } y \in(0,1] \\ \frac{1}{4 y} & \text { if }(\omega, \widetilde{x}) \in[0,1] \times\left(L_{X} \backslash D_{i}\right) \text { and } y \in(0,1] \\ 0 & \text { if }(\omega, \widetilde{x}) \in[0,1] \times L_{X} \text { and } y=1\end{cases}
$$

Then, the correspondence $(\omega, \widetilde{x}) \rightarrow\left(A_{i}(\omega, \widetilde{x}) a_{a_{i}(\widetilde{x})}:[0,1] \times L_{X} \rightarrow 2^{[0,1]}\right.$ is defined by

$$
\left(A_{i}(\omega, \widetilde{x})\right)_{a_{i}(\widetilde{x})}= \begin{cases}\left(0, \frac{9}{10}\right) & \text { if }(\omega, \widetilde{x}) \in[0,1] \times D_{i} ; \\ \left(0, \frac{1}{2}\right] & \text { otherwise }\end{cases}
$$

For each $\omega \in[0,1]$, it has weakly open lower sections in $L_{X}$, and it has a measurable graph.

For each $(\omega, \widetilde{x}) \in[0,1] \times L_{X},\left(A_{i}(\omega, \widetilde{x})\right)_{a_{i}(\widetilde{x})}$ is convex and with nonempty interior in $[0,1]$.

For each $\omega \in[0,1]$, the correspondence $\widetilde{x} \rightarrow \operatorname{cl}\left(A_{i}(\omega, \widetilde{x})\right)_{a_{i}(\widetilde{x})}: L_{X} \rightarrow 2^{[0,1]}$ is upper semicontinuous and nonempty-valued.

For each $\omega \in[0,1]$, let $G_{i}(\omega, \cdot): \mathbb{R} \rightarrow \mathcal{F}(\mathbb{R})$, and let $g_{i}: \mathbb{R} \rightarrow(0,1]$ be such that

$$
\begin{aligned}
& g_{i}(y)=\left\{\begin{array}{ll}
\frac{1}{4} & \text { if } y \in\left(-\infty, \frac{1}{2}\right] ; \\
\frac{3}{4} & \text { if } y \in\left(\frac{1}{2}, \infty\right)
\end{array}\right. \text { and } \\
& G_{i}(\omega, y)(z)= \begin{cases}\frac{1}{2} & \text { if } y \in\left(-\infty, \frac{1}{2}\right] \text { and } z=0 ; \\
1 & \text { if } y \in\left(\frac{1}{2}, \infty\right) \text { and } z \in\{y, y+1\} ; \\
0 & \text { otherwise. }\end{cases}
\end{aligned}
$$

Then, for each $\omega \in[0,1],\left(G_{i}(\omega, \cdot)\right)_{g_{i}(\cdot)}: \mathbb{R} \rightarrow 2^{\mathbb{R}}$ is defined by

$$
\left(G_{i}(\omega, y)\right)_{g_{i}(y)}=\left\{\begin{array}{ll}
\{0\} & \text { if } y \leq \frac{1}{2} ; \\
\{y, y+1\} & \text { if } y>\frac{1}{2}
\end{array} \quad \text { for each }(\omega, y) \in[0,1] \times \mathbb{R} .\right.
$$

For each $\omega \in[0,1],\left(G_{i}(\omega, \cdot)\right)_{g_{i}(\cdot)}: \mathbb{R} \rightarrow 2^{\mathbb{R}}$ is monotone with nonempty values and lower semicontinuous.

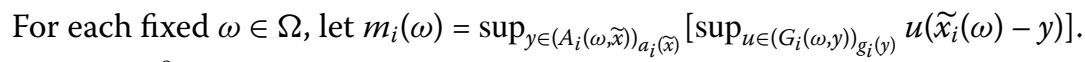

If $\tilde{x}_{i}(\omega) \geq \frac{9}{10}, m_{i}(\omega)>0$. 


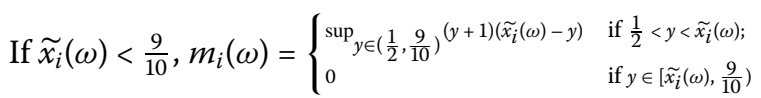

Therefore, if $\frac{1}{2}<\widetilde{x_{i}}(\omega)<\frac{9}{10}, m_{i}(\omega)=\sup _{y \in\left(\frac{1}{2}, \widetilde{x}_{i}(\omega)\right)}(y+1)\left(\widetilde{x_{i}}(\omega)-y\right)>0$ and if $0<\widetilde{x_{i}}(\omega) \leq \frac{1}{2}$, $m_{i}(\omega)=0$.

Consequently, $m_{i}(\omega)>0$ for each $\widetilde{x_{i}}(\omega) \in\left(\frac{1}{2}, 1\right]$.

Then, for each $\omega \in[0,1]$, the set

$$
\begin{aligned}
M_{i}(\omega) & =\left\{\widetilde{x} \in L_{X}: \sup _{y \in\left(A_{i}(\omega, \widetilde{x})\right)_{a_{i}(\tilde{x})}}\left[\sup _{u \in\left(G_{i}(\omega, y)\right)_{g_{i}(y)}} u\left(\widetilde{x_{i}}(\omega)-y\right)\right]>0\right\} \\
& =\left\{\widetilde{x} \in D_{i}: \widetilde{x_{i}}(\omega) \in\left(\frac{1}{2}, 1\right]\right\}
\end{aligned}
$$

is weakly open in $L_{X}$ and $M_{i} \in \mathcal{F} \otimes B\left(L_{X}\right)$.

There exists $\widetilde{x}^{*} \in L_{X}$ such that, for each $i \in I, \widetilde{x}_{i}^{*}(\omega)=0$ for each $\omega \in[0,1]$ and $\mu$-a.e.

(i) $\widetilde{x}_{i}^{*}(\omega) \in \operatorname{cl}\left(\left(A_{i}\left(\omega, \widetilde{x}^{*}\right)\right)_{a_{i}\left(\widetilde{x}^{*}\right)}\right)$;

(ii) $\sup _{u \in\left(G_{i}\left(\omega, \widetilde{x}^{*}(\omega)\right)\right)_{i}\left(\tilde{x}^{*}(\omega)\right)} \operatorname{Re}\left\langle u, \widetilde{x}_{i}^{*}(\omega)-y\right\rangle \leq 0$ for all $y \in\left(A_{i}\left(\omega, \widetilde{x}^{*}\right)\right)_{a_{i}\left(\widetilde{x}^{*}\right)}$.

If $|\mathrm{I}|=1$, we obtain the following corollary of Theorem 3 .

Corollary 2 Let $(\Omega, \mathcal{F}, \mu)$ be a complete finite separable measure space, and let $Y$ be a separable Banach space. Suppose that the following conditions are satisfied.

(A.1) (a) $X: \Omega \rightarrow \mathcal{F}(Y)$ is such that $\omega \rightarrow(X(\omega))_{z}: \Omega \rightarrow 2^{Y}$ is a nonempty convex weakly compact-valued and integrably bounded correspondence;

(b) $X: \Omega \rightarrow \mathcal{F}(Y)$ is such that $\omega \rightarrow(X(\omega))_{z}: \Omega \rightarrow 2^{Y}$ is $\mathcal{F}$-lower measurable;

(A.2) (a) For each $(\omega, \widetilde{x}) \in \Omega \times L_{X},(A(\omega, \widetilde{x}))_{a(\widetilde{x})}$ is convex and has a nonempty interior in the relative norm topology of $(X(\omega))_{z}$;

(b) the correspondence $(\omega, \widetilde{x}) \rightarrow(A(\omega, \widetilde{x}))_{a(\tilde{x})}: \Omega \times L_{X} \rightarrow 2^{Y}$ has a measurable graph, i.e., $\left\{(\omega, \tilde{x}, y) \in \Omega \times L_{X} \times Y: y \in(A(\omega, \widetilde{x}))_{a(\tilde{x})}\right\} \in \mathcal{F} \otimes \beta_{w}\left(L_{X}\right) \otimes \beta(Y)$, where $\beta_{w}\left(L_{X}\right)$ is the Borel $\sigma$-algebra for the weak topology on $L_{X}$ and $\beta(Y)$ is the Borel $\sigma$-algebra for the norm topology on $Y$;

(c) the correspondence $(\omega, \widetilde{x}) \rightarrow(A(\omega, \widetilde{x}))_{a(\tilde{x})}$ has weakly open lower sections, i.e., for each $\omega \in \Omega$ and for each $y \in Y$, the set $\left((A(\omega, \widetilde{x}))_{a(\widetilde{x})}\right)^{-1}(\omega, y)=\left\{\widetilde{x} \in L_{X}: y \in(A(\omega, \widetilde{x}))_{a(\widetilde{x})}\right\}$ is weakly open in $L_{X} ;$

(d) For each $\omega \in \Omega, \widetilde{x} \rightarrow \operatorname{cl}(A(\omega, \widetilde{x}))_{a(\tilde{x})}: L_{X} \rightarrow 2^{Y}$ is upper semicontinuous in the sense that the set $\left\{\tilde{x} \in L_{X}: \operatorname{cl}(A(\omega, \widetilde{x}))_{a(\widetilde{x})} \subset V\right\}$ is weakly open in $L_{X}$ for every norm open subset $V$ of $Y$;

(A.3) $G: \Omega \times Y \rightarrow \mathcal{F}\left(Y^{\prime}\right)$ and $g: Y \rightarrow(0,1]$ are such that:

(a) for each $\omega \in \Omega, y \rightarrow(G(\omega, y))_{g(y)}: Y \rightarrow 2^{Y^{\prime}}$ is monotone with nonempty values;

(b) for each $\omega \in \Omega, y \rightarrow(G(\omega, y))_{g(y)}: L \cap Y \rightarrow 2^{Y^{\prime}}$ is lower semicontinuous from the relative topology of $Y$ into the weak ${ }^{*}$-topology $\sigma\left(Y^{\prime}, Y\right)$ of $Y^{\prime}$ for each one-dimensional flat $L \subset Y$;

(A.4) (a) for each fixed $\omega \in \Omega$, the set $\left\{\tilde{x} \in S_{X}^{1}: \sup _{y \in(A(\omega, \tilde{x}))_{a(\tilde{x})}}\left[\sup _{u \in(G(\omega, y))_{g(y)}} \operatorname{Re}\langle u, \tilde{x}-y\rangle\right]>0\right\}$ is weakly open in $L_{X} ;$

(b) $\left\{(\omega, \tilde{x}): \sup _{u \in(G(\omega, y))_{g(v)}} \operatorname{Re}\langle u, \tilde{x}-y\rangle>0\right\} \in \mathcal{F} \otimes B\left(L_{X}\right)$.

Then, there exists $\widetilde{x}^{*} \in L_{X}$ such that $\mu$-a.e.:

(i) $\widetilde{x}^{*}(\omega) \in \operatorname{cl}\left(A\left(\omega, \widetilde{x}^{*}\right)\right)_{a\left(\widetilde{x}^{*}\right)}$;

(ii) $\sup _{u \in\left(G\left(\omega, \widetilde{x}^{*}(\omega)\right)\right)_{g\left(\tilde{x}^{*}(\omega)\right)}} \operatorname{Re}\left\langle u, \widetilde{x}^{*}(\omega)-y\right\rangle \leq 0$ for all $y \in\left(\left(A\left(\omega, \widetilde{x}^{*}\right)\right)_{a\left(\widetilde{x}^{*}\right)}\right)$. 
We obtain the following random fixed point theorem by using a similar kind of proof as in the case of Theorem 1. This result is a generalization of Browder fixed point theorem [32].

Theorem 4 Let $(\Omega, \mathcal{F}, \mu)$ be a complete finite separable measure space, and let $Y$ be a separable Banach space. Suppose that the following conditions are satisfied.

For each $i \in I$ :

(A.1) (a) $X_{i}: \Omega \rightarrow \mathcal{F}(Y)$ is such that $\omega \rightarrow\left(X_{i}(\omega)\right)_{z_{i}}: \Omega \rightarrow 2^{Y}$ is a nonempty convex weakly compact-valued and integrably bounded correspondence;

(b) $X_{i}: \Omega \rightarrow \mathcal{F}(Y)$ is such that $\omega \rightarrow\left(X_{i}(\omega)\right)_{z_{i}}: \Omega \rightarrow 2^{Y}$ is $\mathcal{F}_{i}$-lower measurable;

(A.2) (a) For each $(\omega, \widetilde{x}) \in \Omega \times L_{X},\left(A_{i}(\omega, \widetilde{x})\right)_{a_{i}(\tilde{x})}$ is convex and has a nonempty interior in the relative norm topology of $\left(X_{i}(\omega)\right)_{z}$;

(b) the correspondence $(\omega, \widetilde{x}) \rightarrow\left(A_{i}(\omega, \widetilde{x})\right)_{a_{i}(\tilde{x})}: \Omega \times L_{X} \rightarrow 2^{Y}$ has a measurable graph, i.e., $\left\{(\omega, \widetilde{x}, y) \in \Omega \times L_{X} \times Y: y \in\left(A_{i}(\omega, \widetilde{x})\right)_{a_{i}(\tilde{x})}\right\} \in \mathcal{F} \otimes \beta_{w}\left(L_{X}\right) \otimes \beta(Y)$, where $\beta_{w}\left(L_{X}\right)$ is the Borel $\sigma$-algebra for the weak topology on $L_{X}$ and $\beta(Y)$ is the Borel $\sigma$-algebra for the norm topology on $Y$;

(c) the correspondence $(\omega, \widetilde{x}) \rightarrow\left(A_{i}(\omega, \widetilde{x})\right)_{a_{i}(\widetilde{x})}$ has weakly open lower sections, i.e., for each $\omega \in \Omega$ and for each $y \in Y$, the set $\left(\left(A_{i}(\omega, \tilde{x})\right)_{a_{i}(\tilde{x})}\right)^{-1}(\omega, y)=\left\{\tilde{x} \in L_{X}: y \in\left(A_{i}(\omega, \widetilde{x})\right)_{a_{i}(\tilde{x})}\right\}$ is weakly open in $L_{X} ;$

Then, there exists $\widetilde{x}^{*} \in L_{X}$ such that for every $i \in I$ and $\mu$-a.e., $\widetilde{x}_{i}^{*}(\omega) \in\left(A_{i}\left(\omega, \widetilde{x}^{*}\right)\right)_{a_{i}\left(\tilde{x}^{*}\right)}$.

If $|\mathrm{I}|=1$, we obtain the following result.

Theorem 5 Let $(\Omega, \mathcal{F}, \mu)$ be a complete finite separable measure space, and let $Y$ be a separable Banach space. Suppose that the following conditions are satisfied.

(A.1) (a) $X: \Omega \rightarrow \mathcal{F}(Y)$ is such that $\omega \rightarrow(X(\omega))_{z}: \Omega \rightarrow 2^{Y}$ is a nonempty convex weakly compact-valued and integrably bounded correspondence;

(b) $X: \Omega \rightarrow \mathcal{F}(Y)$ is such that $\omega \rightarrow(X(\omega))_{z}: \Omega \rightarrow 2^{Y}$ is $\mathcal{F}$-lower measurable;

(A.2) (a) For each $(\omega, \widetilde{x}) \in \Omega \times L_{X},(A(\omega, \widetilde{x}))_{a(\widetilde{x})}$ is convex and has a nonempty interior in the relative norm topology of $(X(\omega))_{z}$;

(b) the correspondence $(\omega, \widetilde{x}) \rightarrow(A(\omega, \widetilde{x}))_{a(\widetilde{x}}: \Omega \times L_{X} \rightarrow 2^{Y}$ has a measurable graph, i.e., $\left\{(\omega, \widetilde{x}, y) \in \Omega \times L_{X} \times Y: y \in(A(\omega, \widetilde{x}))_{a_{i}(\tilde{x})}\right\} \in \mathcal{F} \otimes \beta_{w}\left(L_{X}\right) \otimes \beta(Y)$, where $\beta_{w}\left(L_{X}\right)$ is the Borel $\sigma$-algebra for the weak topology on $L_{X}$ and $\beta(Y)$ is the Borel $\sigma$-algebra for the norm topology on $Y$;

(c) the correspondence $(\omega, \widetilde{x}) \rightarrow(A(\omega, \widetilde{x}))_{a(\widetilde{x})}$ has weakly open lower sections, i.e., for each $\omega \in \Omega$ and for each $y \in Y$, the set $\left((A(\omega, \widetilde{x}))_{a(\tilde{x})}\right)^{-1}(\omega, y)=\left\{\widetilde{x} \in L_{X}: y \in(A(\omega, \widetilde{x}))_{a_{i}(\tilde{x}}\right\}$ is weakly open in $L_{X} ;$

Then, there exists $\widetilde{x}^{*} \in L_{X}$ such that $\widetilde{x}^{*}(\omega) \in\left(A\left(\omega, \widetilde{x}^{*}\right)\right)_{a\left(\widetilde{x}^{*}\right)}$ M-a.e.

Example 4 Let $\Omega=[0,1], \mathcal{F}=\beta([0,1], \mu), Y=\mathbb{R}, I=\{1,2, \ldots, n\}$.

For each $i \in\{1,2, \ldots, n\}$ let us define the following mathematical objects.

Let $X_{i}, L_{X_{i}}$ and $L_{X}$ be defined as in Example $1 . \mathcal{M}_{i}=\left\{\left[0, \frac{1}{2}\right),\left[\frac{1}{2}, \frac{2}{3}\right), \ldots,\left[\frac{i-1}{i}, 1\right]\right\}$ and $\mathcal{F}_{i}=$ $\sigma\left(\mathcal{M}_{i}\right)$.

$C_{i}=\left\{\widetilde{x}_{i}:[0,1] \rightarrow[0,1]: \widetilde{x}_{i}(\omega)=\left\{\begin{array}{ll}\widetilde{x}_{i} & \text { if } \omega \in\left[\frac{i-1}{i}, 1\right] ; \\ 0 & \text { otherwise, }\end{array}\right.\right.$ where $c_{\widetilde{x}_{i}} \in[0,1]$ is constant $\}$ and $D_{i}=$ $\prod_{j \neq i} L_{X_{j}} \times C_{i}$.

We notice that if $x_{i} \in C_{i}$, then it is $\mathcal{F}_{i}$-measurable and $\mu$-integrable, then $C_{i} \subset L_{X_{i}}$ and $C_{i}$ is weakly closed in $L_{X_{i}}$. 
The random fuzzy constraint function $a_{i}: L_{X} \rightarrow(0,1]$ is defined by

$$
a_{i}(\widetilde{x})=\frac{1}{3} \quad \text { for each } \tilde{x} \in L_{X}
$$

For each $\omega \in[0,1]$, the random fuzzy constraint mapping of agent $i, A_{i}(\omega, \cdot): L_{X} \rightarrow \mathcal{F}(\mathbb{R})$ is defined by

$$
A_{i}(\omega, \widetilde{x})(y)= \begin{cases}\frac{\widetilde{x}_{i}(\omega)+2}{5(y+1)} & \text { if }(\omega, \widetilde{x}) \in[0,1] \times D_{i} \text { and } y \in(0,1] \\ 0 & \text { otherwise. }\end{cases}
$$

Then, the correspondence $(\omega, \widetilde{x}) \rightarrow\left(A_{i}(\omega, \widetilde{x})\right)_{a_{i}(\widetilde{x})}:[0,1] \times L_{X} \rightarrow 2^{[0,1]}$ is defined by

$$
\begin{aligned}
\left(A_{i}(\omega, \tilde{x})\right)_{a_{i}(\widetilde{x})} & \left\{y \in[0,1]: A_{i}(\omega, \widetilde{x})(y)>a_{i}(\widetilde{x})\right\} \\
& = \begin{cases}{\left[0, \frac{1+3 \widetilde{x}_{i}(\omega)}{5}\right)} & \text { if }(\omega, \widetilde{x}) \in[0,1] \times D_{i} ; \\
{[0,1]} & \text { otherwise. }\end{cases}
\end{aligned}
$$

It has weakly open lower sections in $L_{X}$, and it has a measurable graph.

For each $(\omega, \tilde{x}) \in[0,1] \times L_{X},\left(A_{i}(\omega, \widetilde{x})\right)_{a_{i}(\widetilde{x})}$ is convex and with a nonempty interior in $[0,1]$.

There exists $\tilde{x}^{*} \in L_{X}$ such that for every $i \in I, \widetilde{x}_{i}^{*}(\omega) \in\left(A_{i}\left(\omega, \widetilde{x}^{*}\right)\right)_{a_{i}\left(\tilde{x}^{*}\right)}$. For instance, let $\tilde{x}^{*}$ such that ${\tilde{x_{1}}}^{*}(\omega)=\frac{1}{3}$ if $\omega \in[0,1]$ and ${\widetilde{x_{i}}}^{*}(\omega)=\left\{\begin{array}{ll}\frac{1}{i+1} & \text { if } \omega \in\left[\frac{i-1}{i}, 1\right] \text {; } \\ 0 & \text { otherwise. }\end{array}\right.$ for each $i \in\{2, \ldots, n\}$.

\section{Appendix}

The results below have been used in the proof of our theorems. For more details and further references see the paper quoted.

Theorem 6 (Projection theorem) Let $(\Omega, \mathcal{F}, \mu)$ be a complete, finite measure space, and let $Y$ be a complete separable metric space. If $H$ belongs to $\mathcal{F} \otimes \beta(Y)$, its projection $\operatorname{Proj}_{\Omega}(H)$ belongs to $\mathcal{F}$.

Theorem 7 (Aumann measurable selection theorem [33]) Let $(\Omega, \mathcal{F}, \mu)$ be a complete finite measure space, let $Y$ be a complete, separable metric space, and let $T: \Omega \rightarrow 2^{Y}$ be a nonempty valued correspondence with a measurable graph, i.e., $G_{T} \in \mathcal{F} \otimes \beta(Y)$. Then there is a measurable function $f: \Omega \rightarrow Y$ such that $f(\omega) \in T(\omega) \mu$-a.e.

Theorem 8 (Diestel's theorem [34], Theorem 3.1) Let $(\Omega, \mathcal{F}, \mu)$ be a complete finite measure space, let $Y$ be a separable Banach space, and let $T: \Omega \rightarrow 2^{Y}$ be an integrably bounded, convex, weakly compact and a nonempty valued correspondence. Then $S_{T}=\left\{x \in L_{1}(\mu, Y)\right.$ : $x(\omega) \in T(\omega) \mu$-a.e. $\}$ is weakly compact in $L_{1}(\mu, Y)$.

Theorem 9 (Carathéodory-type selection theorem [25]) Let $(\Omega, \mathcal{F}, \mu)$ be a complete measure space, let $Z$ be a complete separable metric space, and let $Y$ be a separable Banach space. Let $X: \Omega \rightarrow 2^{Y}$ be a correspondence with a measurable graph, i.e., $G_{X} \in \mathcal{F} \otimes \beta(Y)$ and let $T: \Omega \times Z \rightarrow 2^{Y}$ be a convex-valued correspondence (possibly empty) with a measurable graph, i.e., $G_{T} \in \mathcal{F} \otimes \beta(Z) \otimes \beta(Y)$, where $\beta(Y)$ and $\beta(Z)$ are the Borel $\sigma$-algebras of $Y$ and $Z$, respectively.

Suppose that

(a) for each $\omega \in \Omega, T(\omega, x) \subset X(\omega)$ for all $x \in Z$. 
(b) for each $\omega \in \Omega, T(\omega, \cdot)$ has open lower sections in $Z$, i.e., for each $\omega \in \Omega$ and $y \in Y$, $T^{-1}(\omega, y)=\{x \in Z: y \in T(\omega, x)\}$ is open in $Z$.

(c) for each $(\omega, x) \in \Omega \times Z$, if $T(\omega, x) \neq \emptyset$, then $T(\omega, x)$ has a nonempty interior in $X(\omega)$.

Let $U=\{(\omega, x) \in \Omega \times Z: T(\omega, x) \neq \emptyset\}$ and for each $x \in Z, U^{x}=\{\omega \in \Omega:(\omega, x) \in U\}$ and for each $\omega \in \Omega, U^{\omega}=\{x \in Z:(\omega, x) \in U\}$. Then for each $x \in Z, U^{x}$ is a measurable set in $\Omega$, and there exists a Caratheodory-type selection from $T_{\mid U}$, i.e., there exists a function $f: U \rightarrow Y$ such that $f(\omega, x) \in T(\omega, x)$ for all $(\omega, x) \in U$, for each $x \in Z, f(\cdot, x)$ is measurable on $U^{x}$ and for each $\omega \in \Omega, f(\omega, \cdot)$ is continuous on $U^{\omega}$. Moreover, $f(\cdot, \cdot)$ is jointly measurable.

Theorem 10 (U.s.c. lifting theorem [33]) Let $Y$ be a separable space, let $(\Omega, \mathcal{F}, \mu)$ be a complete finite measure space, and let $X: \Omega \rightarrow 2^{Y}$ be an integrably bounded, nonempty, convex valued correspondence such that for all $\omega \in \Omega, X(\omega)$ is a weakly compact, convex subset of $Y$. Denote by $S_{X}$ the set $\left\{x \in L_{1}(\mu, Y): x(\omega) \in X(\omega) \mu\right.$-a.e. $\}$. Let $T: \Omega \times S_{X} \rightarrow 2^{Y}$ be a nonempty, closed, convex-valued correspondence such that $T(\omega, x) \subset X(\omega)$ for all $(\omega, x) \in$ $\Omega \times S_{X}^{1}$. Assume that for each fixed $x \in S_{X}, T(\cdot, x)$ has a measurable graph, and that for each fixed $\omega \in \Omega, T(\omega, \cdot): S_{X} \rightarrow 2^{Y}$ is u.s.c. in the sense that the set $\left\{x \in S_{X}: T(\omega, x) \subset V\right\}$ is weakly open in $S_{X}$ for every norm open subset $V$ of $Y$. Define the correspondence $\Phi: S_{X} \rightarrow$ $2^{S_{X}}$ by

$$
\Phi(x)=\left\{y \in S_{X}: y(\omega) \in T(\omega, x) \mu \text {-a.e. }\right\} .
$$

Then $\Phi$ is weakly u.s.c., i.e., the set $\left\{x \in S_{X}: \Phi(x) \subset V\right\}$ is weakly open in $S_{X}$ for every weakly open subset $V$ of $S_{X}$.

\section{Competing interests}

The author declares that they have no competing interests.

\section{Acknowledgements}

The author would like to thank two referees for helpful comments and suggestions.

Received: 7 May 2013 Accepted: 25 July 2013 Published: 9 August 2013

\section{References}

1. Kim, WK, Lee, KH: Fuzzy fixed point and existence of equilibria of fuzzy games. J. Fuzzy Math. 6, 193-202 (1998)

2. Patriche, M: Equilibrium in Games and Competitive Economies. The Publishing House of the Romanian Academy, Bucharest (2011)

3. Debreu, G: A social equilibrium existence theorem. Proc. Natl. Acad. Sci. USA 38, 886-903 (1952)

4. Shafer, W, Sonnenschein, H: Equilibrium in abstract economies without ordered preferences. J. Math. Econ. 2, 345-348 (1975)

5. Yannelis, NC, Prabhakar, ND: Existence of maximal elements and equilibrium in linear topological spaces. J. Math Econ. 12, 233-245 (1983)

6. Akinlar, M: Application of a finite element method for variational inequalities. J. Inequal. Appl. 2013, 45 (2013)

7. Balooee, J, Cho, YJ: Perturbed projection and iterative algorithms for a system of general regularized nonconvex variational inequalities. J. Inequal. Appl. 2012, 141 (2012)

8. Fang, C, Chen, S, Yang, C: An algorithm for solving multi-valued variational inequality. J. Inequal. Appl. 2013, 218 (2013)

9. Hung, N: Existence conditions for symmetric generalized quasi-variational inclusion problems. J. Inequal. Appl. 2013, 40 (2013)

10. Noor, MA: Some aspects of extended general variational inequalities. Abstr. Appl. Anal. 2012, Article ID 303569 (2012)

11. Noor, MA, Noor, Kl: Some new classes of extended general mixed quasi-variational inequalities. Abstr. Appl. Anal. 2012, Article ID 962978 (2012)

12. Noor, MA, Elsanousi, SA: Iterative algorithms for random variational inequalities. Panam. Math. J. 3, 39-50 (1993)

13. Cho, YJ, Huang, NJ, Kang, SM: Random generalized set-valued strongly nonlinear implicit quasi-variational inequalities. J. Inequal. Appl. 5, 515-531 (2000)

14. Gwinner, J, Raciti, F: On a class of random variational inequalities on random sets. Numer. Funct. Anal. Optim. 27(5-6), 619-636 (2006)

15. Gwinner, J, Raciti, F: On monotone variational inequalities with random data. J. Math. Inequal. 3(3), 443-453 (2009) 
16. Gwinner, J, Raciti, F: Some equilibrium problems under uncertainty and random variational inequalities. Ann. Oper. Res. 200, 299-319 (2012)

17. Lan, HY: Projection iterative approximations for a new class of general random implicit quasi-variational inequalities. J. Inequal. Appl. 2006, Article ID 81261 (2006)

18. Nazemi, SS: A new system of random generalized variational inclusions with random fuzzy mappings and random $(H(\cdot . \cdot) \Phi)$ - $\eta$-accretive mappings in Banach spaces. SRN Appl. Math. 2012, Article ID 731058 (2012). doi:10.5402/2012/731058

19. Tan, KK, Tarafdar, E, Yuan, GX-Z: A study of variational inequalities for set-valued mappings. J. Inequal. Appl. 3, 161-181 (1991)

20. Tan, K-K, Yuan, GX-Z: Random equilibria of random generalized games with applications to non-compact random quasi-variational inequalities. Topol. Methods Nonlinear Anal. 5, 59-82 (1995)

21. Yuan, GX-Z, Roy, J-M: Some new deterministic and random variational inequalities and their applications. J. Appl. Math. Stoch. Anal. 8(4), 381-391 (1995)

22. Yuan, GX-Z: The study of minimax inequalities and applications to economies and variational inequalities. Mem. Am. Math. Soc. 132, 625 (1988)

23. Aubin, JP, Frankowska, H: Set-Valued Analysis. Birkhäuser, Basel (1990)

24. Debreu, G: Integration of correspondences. In: Proc. Fifth Berkeley Symp. Math. Statist. Prob., vol. 2, pp. $351-372$. University of California Press, Berkeley (1966)

25. Kim, T, Prikry, K, Yannelis, NC: On a Carathéodory-type selection theorem. J. Math. Anal. Appl. 135, 664-670 (1988)

26. Zadeh, LA: Fuzzy sets. Inf. Control 8, 338-353 (1965)

27. Chang, SS: Coincidence theorems and variational inequalities for fuzzy mappings. Fuzzy Sets Syst. 61, 359-368 (1994)

28. Uea, NO, Kumam, P: A generalized nonlinear random equations with random fuzzy mappings in uniformly smooth Banach spaces. J. Inequal. Appl. 2010, Article ID 728452 (2010). doi:10.1155/2010/728452

29. Patriche, M: Bayesian abstract economies. Optimization 60(4), 451-461 (2011)

30. Fan, K: Fixed-point and minimax theorems in locally convex topological linear spaces. Proc. Natl. Acad. Sci. USA 38, $121-126(1952)$

31. Castaing, C, Valadier, M: Convex Analysis and Measurable Multifunctions. Lecture Notes in Mathematics, vol. 580. Springer, New York (1977)

32. Browder, FE: The fixed point theory of multi-valued mappings in topological vector spaces. Math. Ann. 177, 283-301 (1968)

33. Yannelis, NC: On the existence of Bayesian Nash equilibrium. In: Agramovich, Y, Avgerinos, E, Yannelis, NC (eds.) Functional Analysis and Economic Theory. Springer, Berlin (1998)

34. Yannelis, NC: Integration of Banach-valued correspondences. In: Khan, MA, Yannelis, NC (eds.) Equilibrium Theory in Infinite Dimensional Spaces. Studies in Economic Theory. Springer, Berlin (1991)

35. Diestel, J, Uhl, J: Vector Measures. Mathematical Surveys, vol. 15. Am. Math. Soc., Providence (1977)

doi:10.1186/1029-242X-2013-374

Cite this article as: Patriche: Equilibrium of Bayesian fuzzy economies and quasi-variational inequalities with random fuzzy mappings. Journal of Inequalities and Applications 2013 2013:374.

\section{Submit your manuscript to a SpringerOpen ${ }^{\odot}$ journal and benefit from:}

- Convenient online submission

Rigorous peer review

- Immediate publication on acceptance

Open access: articles freely available online

- High visibility within the field

- Retaining the copyright to your article 